Nevada

Environmental

Restoration

Project

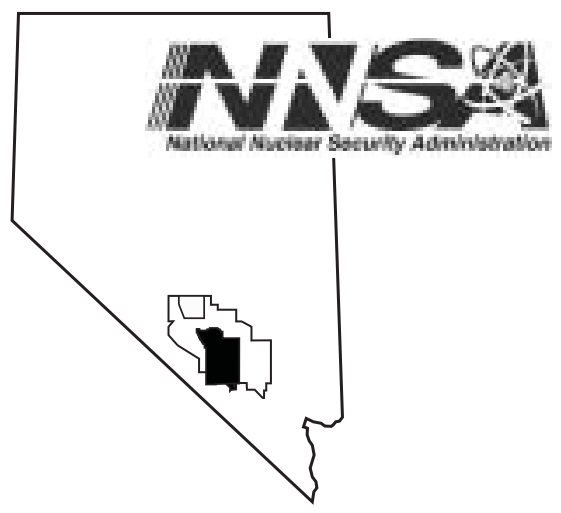

Closure Report for Corrective

Action Unit 346: Areas 8, 10 Housekeeping Sites, Nevada Test Site, Nevada

Controlled Copy No.:

Revision: 0

August 2003

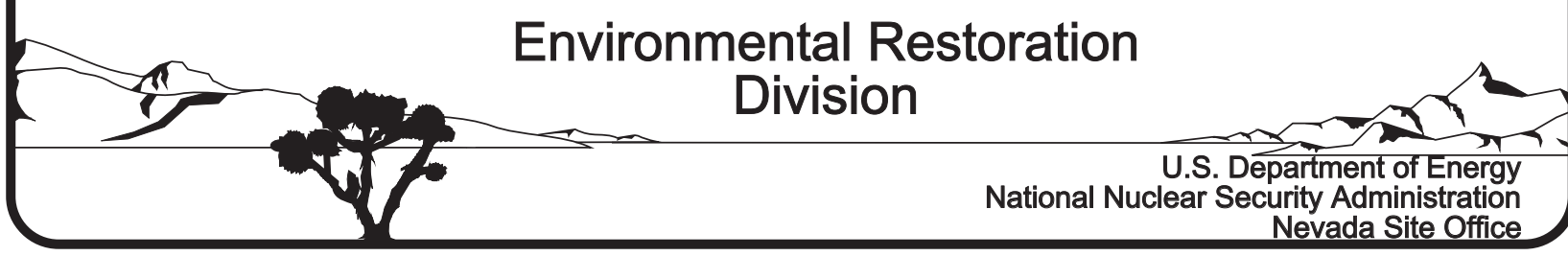




\section{DISCLAIMER STATEMENT}

Reference herein to any specific commercial product, process, or service by trade name, trademark, manufacturer, or otherwise, does not necessarily constitute or imply its endorsement, recommendation, or favoring by the U.S. Government or any agency thereof or its contractors or subcontractors.

\section{AVAILABILITY STATEMENT}

Available for sale to the public from-

U.S. Department of Commerce

National Technical Information Service

5285 Port Royal Road

Springfield, VA 22161-0002

Telephone: 800.553 .6847

Fax: 703.605.6900

E-mail: orders@ntis.fedworld.gov

Online ordering: http://www.ntis.gov/ordering.htm

Available electronically at http://www.doe.gov/bridge

Available for a processing fee to U.S. Department of Energy and its contractors, in paper, from-

U.S. Department of Energy

Office of Scientific and Technical Information

P.O. Box 62

Oak Ridge, TN 37831-0062

Telephone: 865.576.8401

Fax: 865.576.5728

E-mail: reports@,adonis.osti.gov 


\title{
CLOSURE REPORT FOR CORRECTIVE ACTION UNIT 346: AREAS 8, 10 HOUSEKEEPING SITES, NEVADA TEST SITE, NEVADA
}

\author{
Prepared for: \\ U.S. Department of Energy \\ National Nuclear Security Administration \\ Nevada Site Office \\ Work Performed Under Contract No. DE-AC 08-96NV11718
}

Controlled Copy No:

Revision: 0

August 2003 
THIS PAGE INTENTIONALLY LEFT BLANK 


\section{CLOSURE REPORT FOR CORRECTIVE ACTION UNIT 346: AREAS 8, 10 HOUSEKEEPING SITES, NEVADA TEST SITE, NEVADA}
Approved by: $\quad$ SIGNATURE APPROVE
Janet Appenzeller-Wing, Project Manager Industrial Sites Project

\footnotetext{
Approved by: SIGNATURE APPROVE

Runore C. Wycoff, Director
}

Environmental Restoration Division
Date: $\quad 8 / 18 / 03$

Date: $\quad 8 / 18 / 03$ 
THIS PAGE INTENTIONALLY LEFT BLANK 


\section{TABLE OF CONTENTS}

ACRONYMS AND ABBREVIATIONS .................................................................... vii

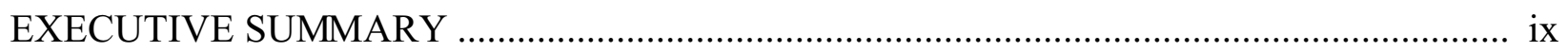

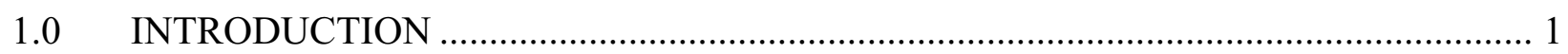

2.0 CLOSURE ACTIVITIES .................................................................................. 3

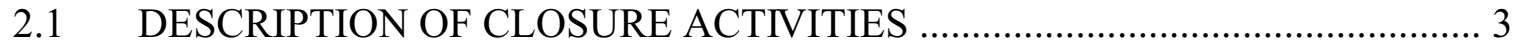

2.1.1 Preplanning and Site Preparation ........................................................... 3

2.2 WASTE CHARACTERIZATION ACTIVITIES .............................................. 3

2.2.1 CAS 10-14-01: Transformer ................................................................. 3

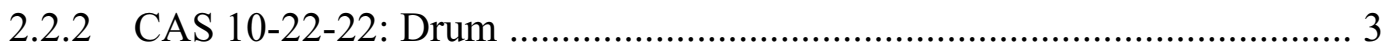

2.2.3 CAS 10-22-25: Drum ............................................................................. 5

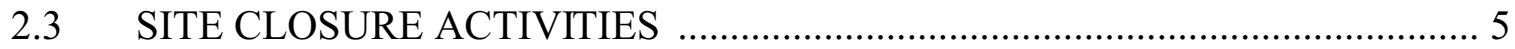

2.3.1 CAS 08-22-04: Drums (2) …................................................................. 5

2.3.2 CAS 08-22-11: Drums; Buckets ............................................................. 5

2.3.3 CAS 08-24-02: Battery .................................................................... 5

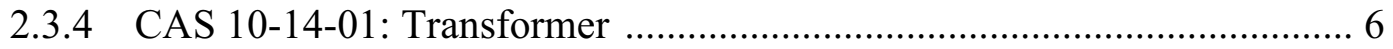

2.3.5 CAS 10-22-06: Drum (Gas Block) ........................................................... 6

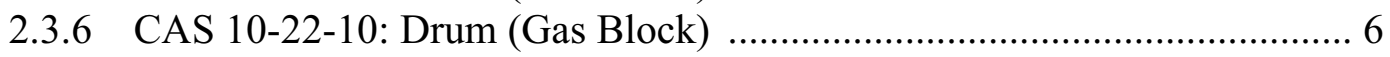

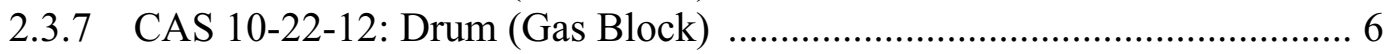

2.3.8 CAS 10-22-13: Drum (Gas Block) ….................................................. 7

2.3.9 CAS 10-22-16: Drum (Gas Block) …............................................... 7

2.3.10 CAS 10-22-22: Drum ............................................................................ 7

2.3.11 CAS 10-22-25: Drum .......................................................................... 7

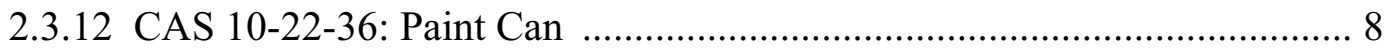

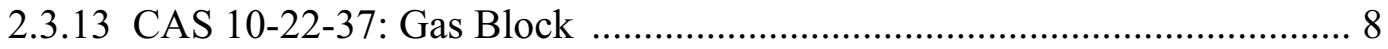

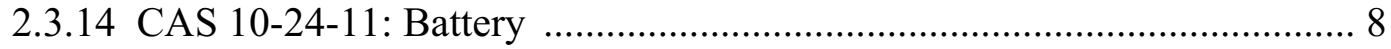

WASTE DISPOSITION ................................................................................ 9

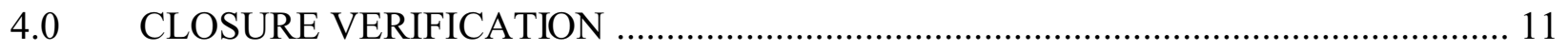

5.0 SUMMARY AND RECOMMENDATIONS ...................................................... 13

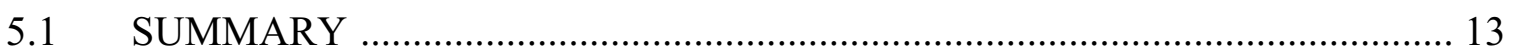

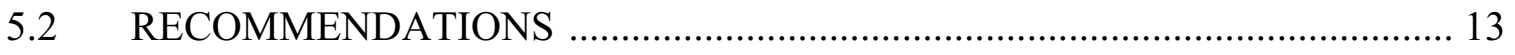

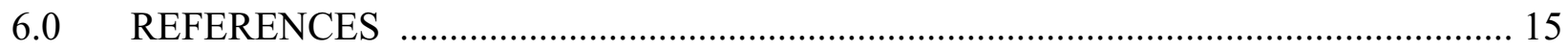

\section{FIGURES}

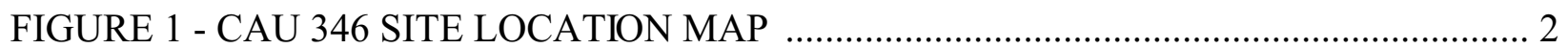




\section{TABLE OF CONTENTS (continued)}

\section{TABLES}

TABLE 1 - SUMMARY OF ANALYTICAL RESULTS FOR WASTE

CHARACTERIZATION SAMPLES

\section{APPENDICES}

APPENDIX A: SAMPLE ANALYTICAL RESULTS

APPENDIX B: SECTORED HOUSEKEEPING SITE CLOSURE VERIFICATION FORMS

APPENDIX C: U.S. DEPARTMENT OF ENERGY NATIONAL NUCLEAR SECURITY ADMINISTRATION NEVADA OPERATIONS OFFICE NEPA ENVIRONMENTAL EVALUATION CHECKLIST

DISTRIBUTION LIST 


\section{ACRONYMS AND ABBREVIATIONS}

\begin{tabular}{|c|c|}
\hline $\mathrm{BN}$ & Bechtel Nevada \\
\hline CAS & Corrective Action Site(s) \\
\hline CAU & Corrective Action Unit \\
\hline $\mathrm{COC}$ & Contaminant(s) of Concern \\
\hline CFR & Code of Federal Regulations \\
\hline $\mathrm{DOE} / \mathrm{NV}$ & U.S. Department of Energy Nevada Operations Office \\
\hline EPA & U.S. Environmental Protection Agency \\
\hline ER & Environmental Restoration \\
\hline FFACO & Federal Facility Agreement and Consent Order \\
\hline gal & Gallon(s) \\
\hline $\mathrm{L}$ & Liter(s) \\
\hline $\mathrm{mg} / \mathrm{kg}$ & Milligram(s) per kilogram \\
\hline $\mathrm{mg} / \mathrm{L}$ & Milligram(s) per liter \\
\hline NAC & Nevada Administrative Code \\
\hline NA & Not Applicable \\
\hline ND & Not Detected \\
\hline NDEP & Nevada Division of Environmental Protection \\
\hline NNSA/NSO & $\begin{array}{l}\text { U.S. Department of Energy, National Nuclear Security Administration Nevada } \\
\text { Site Office }\end{array}$ \\
\hline NTS & Nevada Test Site \\
\hline PA & Preliminary Assessment \\
\hline PCBs & polychlorinated biphenyls \\
\hline $\mathrm{pCi} / \mathrm{g}$ & picoCuries per gram \\
\hline ppm & parts per million \\
\hline
\end{tabular}




\section{ACRONYMS AND ABBREVIATIONS (continued)}

RCT Radiological Control Technician

RCRA Resource Conservation and Recovery Act

SVOC Semivolatile Organic Compound(s)

SWO Solid Waste Operations

TCLP Toxicity Characteristic Leaching Procedure

TPH Total Petroleum Hydrocarbons

VOC Volatile Organic Compound(s) 


\section{EXECUTIVE SUMMARY}

The 14 Corrective Action Sites (CASs) included in Corrective Action Unit 346 were closed by the following actions:

CAS 08-22-04: Clean-closed with no action necessary

CAS 08-22-11: Clean-closed

CAS 08-24-02: Clean-closed with no action necessary

CAS 10-14-01: Clean-closed

CAS 10-22-06: Clean-closed

CAS 10-22-10: Clean-closed

CAS 10-22-12: Clean-closed

CAS 10-22-13: Clean-closed

CAS 10-22-16: Clean-closed

CAS 10-22-22: Clean-closed

CAS 10-22-25: Clean-closed

CAS 10-22-36: Clean-closed

CAS 10-22-37: Clean-closed

CAS 10-24-11: Clean-closed 
CLOSURE RE PORT - CAU 346

Section: Executive Sum mary

Revision : 0

Date: August 2003

THIS PAGE INTENTIONALLY LEFT BLANK 


\subsection{INTRODUCTION}

This Closure Report documents the closure activities conducted for Corrective Action Unit (CAU) 346: Areas 8, 10 Housekeeping Sites. CAU 346 is listed in Appendix III of the Federal Facility Agreement and Consent Order (FFACO, 1996) and consists of the following 14 Corrective Action Sites (CASs) located in Areas 8 and 10 of the Nevada Test Site (NTS) (see Figure 1):

$\begin{array}{ll}\text { CAS 08-22-04: } & \text { Drums (2) } \\ \text { CAS 08-22-11: } & \text { Drums; Bucket } \\ \text { CAS 08-24-02: } & \text { Battery } \\ \text { CAS 10-14-01: } & \text { Transformer } \\ \text { CAS 10-22-06: } & \text { Drum (Gas Block) } \\ \text { CAS 10-22-10: } & \text { Drum (Gas Block) } \\ \text { CAS 10-22-12: } & \text { Drum (Gas Block) } \\ \text { CAS 10-22-13: } & \text { Drum (Gas Block) } \\ \text { CAS 10-22-16: } & \text { Drum (Gas Block) } \\ \text { CAS 10-22-22: } & \text { Drum } \\ \text { CAS 10-22-25: } & \text { Drum } \\ \text { CAS 10-22-36: } & \text { Paint Can } \\ \text { CAS 10-22-37: } & \text { Gas Block } \\ \text { CAS 10-24-11: } & \text { Battery }\end{array}$

Closure activities consisted of closing each CAS by removing debris and/or material, disposing of the generated waste, and verifying that each site was clean-closed by visual inspection and/or laboratory analysis of soil verification samples.

Copies of the analytical results for the site verification samples are included in Appendix A. Copies of the Sectored Housekeeping Site Closure Verification Forms for each of the 14 CASs are included in Appendix B. 


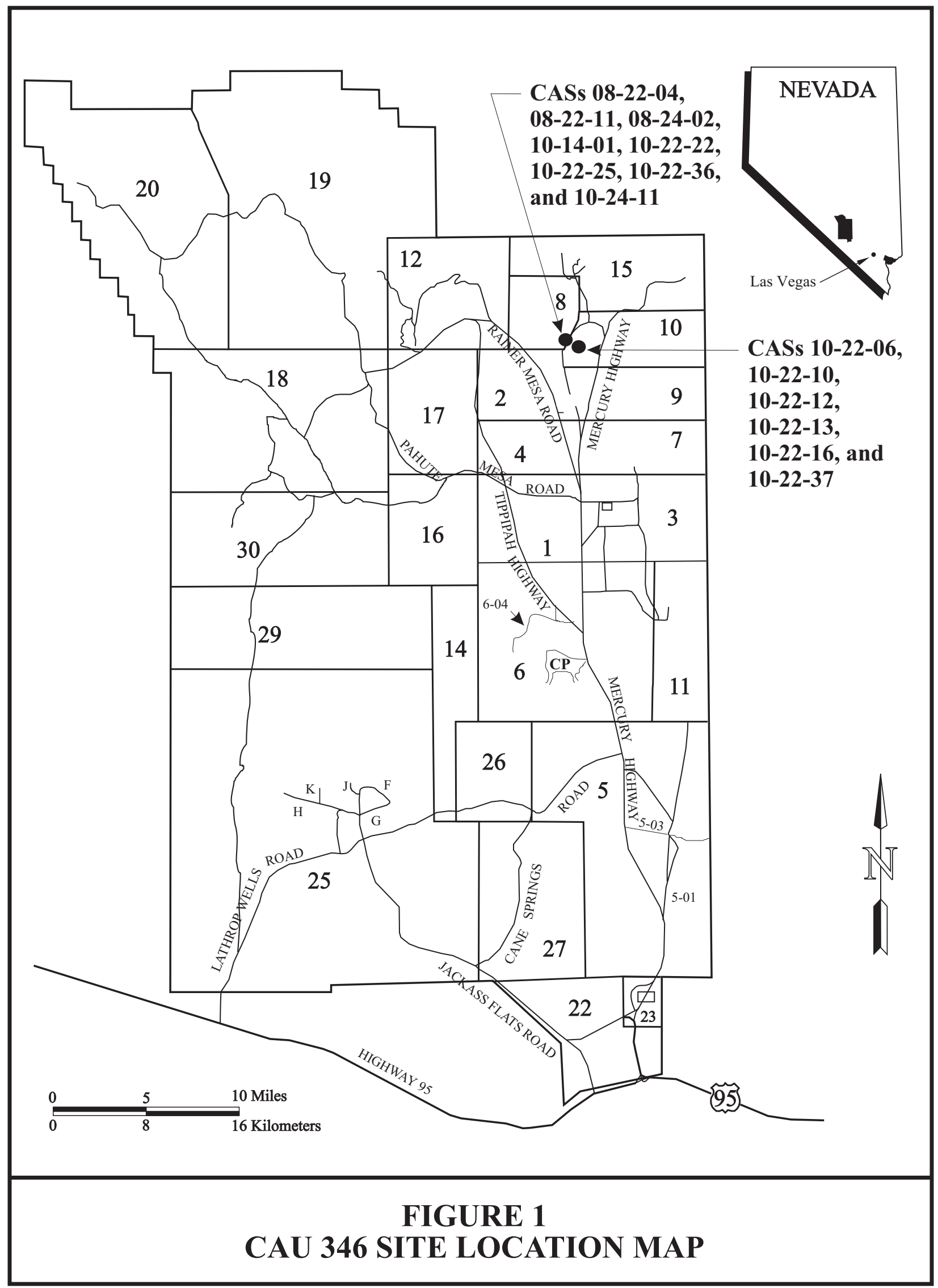




\subsection{CLOSURE ACTIVITIES}

This section details the specific corrective action activities completed during the closure of CAU 346: Areas 8, 10 Housekeeping Sites.

\subsection{DESCRIPTION OF CLOSURE ACTIVITIES}

\subsubsection{Preplanning and Site Preparation}

Planning documents prepared prior to the beginning of closure activities included the following:

Sectored Clean-up Work Plan For Housekeeping Category Waste Sites (U.S. Department of Energy, National Nuclear Security Administrations Nevada Site Office [NNSA/NSO], 2003).

Generic Field Management Plan for Housekeeping Category Waste Sites (Bechtel Nevada [BN], 2000).

Site-Specific Health and Safety Plan for Corrective Action Units 176, 346, 350, 351, 352, \& 523 Housekeeping Sites, Nevada Test Site, Nevada (BN, 2003).

BN Solid Waste Operations (SWO) Work Packages.

NNSA/NSO Real Estate/Operation Permit.

BN Environmental Restoration (ER) and SWO personnel held a pre-job field briefing on May 29, 2003 prior to the start of site-closure field activities.

\subsection{WASTE CHARA CTERIZATION ACTIVITIES}

At CASs 10-14-01, 10-22-22, and 10-22-25, samples were collected and analyzed to characterize the waste that was expected to be generated during site-closure activities. Table 1 provides the results of analyses that were done for the waste characterization samples. Appendix A provides the analytical reports for the waste characterization samples.

\subsubsection{CAS 10-14-01: Transformer}

An oil-filled circuit breaker was located at this site. The circuit breaker contained three separate oil reservoirs which were each sampled for polychlorinated biphenyls (PCBs). Analytical results showed that the circuit breaker is non-PCB electrical equipment as defined by Title 40, Code of Federal Regulation (CFR) Part 761 (CFR, 2002).

\subsubsection{CAS 10-22-22: Drum}

One 151-liter (L) (40-gallon [gal]) drum containing granular dehumidifying crystals was located at this site. The crystals were sampled and analyzed for Toxicity Characteristic Leaching 
Procedure (TCLP) volatile organic compounds (VOCs), TCLP semivolatile organic compounds (SVOCs), TCLP Resource Conservation and Recovery Act (RCRA) metals, total petroleum hydrocarbons (TPH), Gamma emitters and Tritium. Analytical results presented in Table 1 show that the contents of the drum was not hazardous waste as defined by Title 40 CFR Part 261.24 (CFR, 2002), TPH waste as defined by Part 445A.2272 of the Nevada Administrative Code (NAC, 2003), or as radioactive waste. Analytical data summaries for the samples are included in Appendix A.

\section{TABLE 1 - SUMMARY OF ANALYTICAL RESULTS FOR WASTE}

\section{CHARACTERIZATION SAMPLES*}

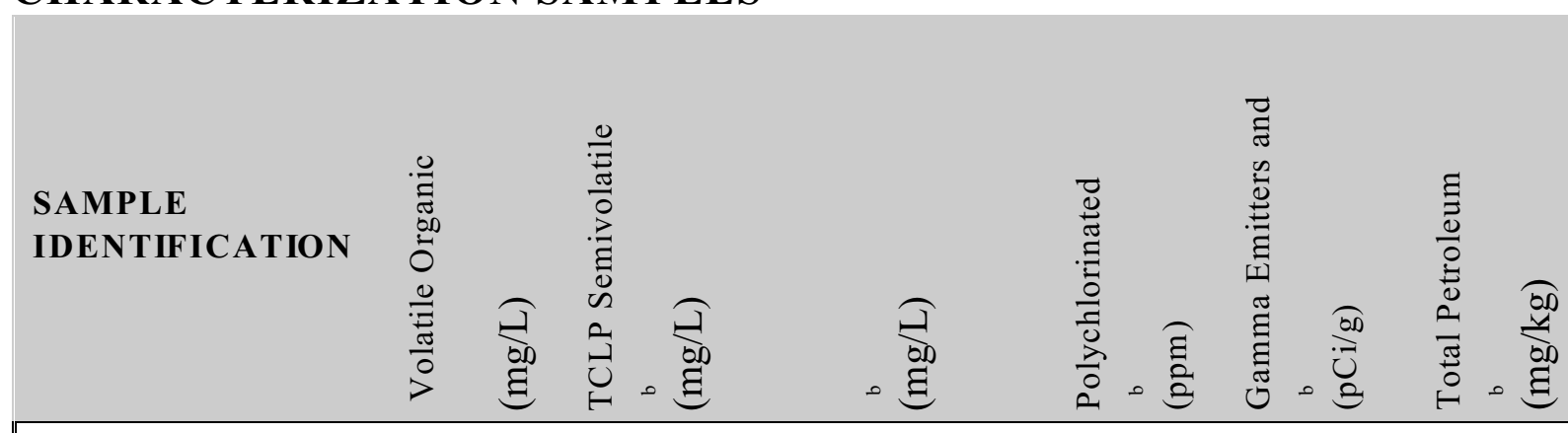

CAS 10-14-01 - Oil-Filled Circuit Breaker (Sample Delivery Group [SDG] V1894)

\begin{tabular}{|c|c|c|c|c|c|c|}
\hline $101401-1$ & NS & NS & NS & ND & NS & NS \\
\hline $101401-2$ & NS & NS & NS & 1.8 & NS & NS \\
\hline $101401-3$ & NS & NS & NS & 1.9 & NS & NS \\
\hline
\end{tabular}

CAS 10-22-22 - Drum (SDG V1889 and V1890)

\begin{tabular}{|l|c|c|ll|l|l|l|}
\hline $102222-01$ & ND & ND & \begin{tabular}{ll|l} 
Barium \\
Cadmium
\end{tabular} & 0.171 & NS & ND & ND \\
\hline
\end{tabular}

CAS 10-22-25 - Drum (SDG V1889 and V1890)

\begin{tabular}{|c|c|c|lr|c|c|c|}
\hline $102225-01$ & ND & ND & $\begin{array}{l}\text { Barium } \\
\text { Cadmium }\end{array}$ & $\begin{array}{l}0.289 \\
0.026\end{array}$ & NS & ND & ND \\
\hline \hline \multirow{2}{*}{$102225-03$} & ND & ND & $\begin{array}{l}\text { Barium } \\
\text { Cadmium }\end{array}$ & $\begin{array}{l}0.225 \\
0.023\end{array}$ & NS & NS & ND \\
\hline
\end{tabular}

* Refer to Appendix A for laboratory data reports.

a Toxicity Characteristic Leaching Procedure

b TCLP VOCs, TCLP SVOCs, TCLP RCRA Metals, PCBs, TPH, Gamma, and Tritium samples analyzed by U.S. Environmental Protection Agency (EPA) Method 1311/8240, 1311/8270, 1311/6010 and 1311/7000, 8082, 8015, 901.1 and 906.0 respectively (EPA, 1996). See analytical reports in Appendix A for detection limits.

${ }^{\mathrm{c}}$ Resource Conservation and Recovery Act.

Definitions:

$\mathrm{mg} / \mathrm{L}=$ Milligram(s) per liter

$\mathrm{mg} / \mathrm{kg}=$ Milligrams(s) per kilogram

NS $=$ Not Sampled

$\mathrm{ND}=$ Not detected at laboratory reporting limit or less than laboratory Minimum Detectable Activity. See Appendix A for laboratory reporting limits.

ppm $=$ parts per million 


\subsubsection{CAS 10-22-25: Drum}

One 151-L (40-gal) drum containing granular dehumidifying crystals was located at this site. The crystals were sampled and analyzed for TCLP VOCs, TCLP SVOCs, TCLP RCRA metals, $\mathrm{TPH}$, Gamma emitters, and Tritium. Analytical results presented in Table 1 show that the contents of the drum was not hazardous waste as defined by Title 40 CFR Part 261.24 (CFR, 2002), TPH waste as defined by Part 445A.2272 of the Nevada Administrative Code (NAC, 2003), or as radioactive waste. Analytical data summaries for the samples are included in Appendix A.

\subsection{SITE CLOSURE ACTIVITIES}

Appendix B provides the Housekeeping Site Closure Verification Forms showing before and after photographs and providing site information.

\subsubsection{CAS 08-22-04: Drums (2)}

The drums originally identified in the FFACO were not present at the time of the Preliminary Assessment (PA) or during the subsequent BN ER site visit (IT, 2002). The original waste site was located near a mud pit associated with the four craters remaining from the U8d crater area and outlying post-shot holes from the 1970 "Baneberry test (U.S. Department of Energy, Nevada Operations Office [DOE/NV], 2000). Site closure was documented with photographs and field notes. No further action is required at this site.

\subsubsection{CAS 08-22-11: Drums; Buckets}

Three empty buckets and two empty drums were located in two mud pits associated with the U8d crater area and outlying post-shot holes from the 1970 "Baneberry test (DOE/NV, 2000). Previous soil sampling results from the PA indicated the presence of diesel range petroleum hydrocarbons above the Nevada State Action Level (Nevada Administrative Code [NAC], 2003) in the northern mud pit known as Mud Pit B (IT, 2002). Mud Pit B is part of CAS 08-09-03 which is currently included in CAU 357 and scheduled for remediation later in 2003. The housekeeping waste was removed from the mud pits. However, soil samples were not collected, as the diesel range TPH in the soil will be addressed as part of CAU 357 closure activities. All CAU 346 closure activities were documented with photographs and field notes. Clean closure of the housekeeping portion of this site was verified by visual inspection. Verification samples were not required of collected. No further actions relating to CAU 346 are required at this site.

\subsubsection{CAS 08-24-02: Battery}

The battery originally identified in the FFACO was not present at the time of the PA or during the subsequent BN ER site visit (IT, 2002). The original waste site was located north of the four craters (U10am1 through U10am4) remaining from the 1969 "Tun-A through "Tun-D detonations (DOE/NV, 2000). Site closure was documented with photographs and field notes. No further action is required at this site. 


\subsubsection{CAS 10-14-01: Transformer}

An oil-filled circuit breaker was located directly south of the U10cb crater from the 1984 "Normanna test (DOE/NV, 2000). The circuit breaker contained three oil reservoirs that were sampled and showed to contain a maximum of 1.9 parts per million PCBs. This classified the circuit breaker as non-PCB electrical equipment as defined by Title 40, Code of Federal Regulation (CFR) Part 761 (CFR, 2002). The circuit breaker was sprayed and cleaned to address Hanta virus concerns and was removed from the site for recycling/disposal. All closure activities were documented with photographs and field notes. Clean closure of this site was verified by visual inspection. Verification samples were not required or collected. No further action is required at this site.

\subsubsection{CAS 10-22-06: Drum (Gas Block)}

One gas block drum was located at the site near the crater from the 1966 "Vigil and "Rovenna tests (DOE/NV, 2000). During the PA a surface soil sample was collected adjacent to the west side of the drum and analytical results showed no COC were present in the soil (IT, 2002). The drum was filled with solidified grout and 27 cables of various sizes were protruding from it. Each of the cables was cut and screened for radioactivity. After the drum was released it was disposed of at the Area 9 U10c Landfill. All closure activities were documented with photographs and field notes. Clean closure of this site was verified by visual inspection. Verification samples were not required or collected. No further actions are required at this site.

\subsubsection{CAS 10-22-10: Drum (Gas Block)}

One partially buried gas block drum was located at the site southwest of the crater from the 1968 "Tub-A and "Tub-F detonations (DOE/NV, 2000). During the PA a surface soil sample was collected adjacent to the south side of the buried drum and analytical results showed no COC were present in the soil (IT, 2002). The drum was filled with solidified grout and 26 cables of various sizes were protruding from it. Each of the cables was cut and screened for radioactivity. After the drum was released it was disposed of at the Area 9 U10c Landfill. All closure activities were documented with photographs and field notes. Clean closure of this site was verified by visual inspection; no verification samples were required or collected. No further actions are required at this site.

\subsubsection{CAS 10-22-12: Drum (Gas Block)}

One gas block drum was located at the site near the a post-shot hole associated with the 1968 "Tub-C detonation (DOE/NV, 2000). During the PA a surface soil sample was collected immediately south of the drum and analytical results showed no COC were present in the soil (IT, 2002). The drum was filled with solidified grout and 21 cables of various sizes were protruding from it. Each of the cables was cut and screened for radioactivity. After the drum was released it was disposed of at the Area 9 U10c Landfill. All closure activities were documented with photographs and field notes. Clean closure of this site was verified by visual inspection. Verification samples were not required or collected. No further actions are required at this site. 


\subsubsection{CAS 10-22-13: Drum (Gas Block)}

One gas block drum was located at the site near the a post-shot hole associated with the 1968 "Tub-C detonation (DOE/NV, 2000). During the PA a surface soil sample was collected adjacent to the south side of the drum and analytical results showed no COC were present in the soil (IT, 2002). The drum was filled with solidified grout and 23 cables of various sizes were protruding from it. Each of the cables was cut and screened for radioactivity. After the drum was released it was disposed of at the Area 9 U10c Landfill. All closure activities were documented with photographs and field notes. Clean closure of this site was verified by visual inspection. Verification samples were not required or collected. No further actions are required at this site.

\subsubsection{CAS 10-22-16: Drum (Gas Block)}

One gas block drum was located at the site directly north of the crater associated with the 1966 "Reo test (DOE/NV, 2000). The drum was filled with solidified grout and 52 cables of various sizes were protruding from it. Each of the cables was cut and screened for radioactivity. After the drum was released it was disposed of at the Area 9 U10c Landfill. All closure activities were documented with photographs and field notes. Clean closure of this site was verified by visual inspection. Verification samples were not required or collected. No further actions are required at this site.

\subsubsection{CAS 10-22-22: Drum}

One 151-L (40-gal) drum filled with dehumidifying crystals was located between the two craters (U10am3 and U10am4) associated with the 1969 "Tun-C and "Tun-D detonations (DOE/NV, 2000). During the PA a surface soil sample was collected beneath the drum and analytical results showed no COC were present in the soil (IT, 2002). The drum was screened for radioactivity. After the drum was released it was disposed of at the Area 9 U10c Landfill. All closure activities were documented with photographs and field notes. Clean closure of this site was verified by visual inspection. Verification samples were not required or collected. No further actions are required at this site.

\subsubsection{CAS 10-22-25: Drum}

One 151-L (40-gal) drum filled with dehumidifying crystals was located between the two craters (U10am1 and U10am2) associated with the 1969 "Tun-A and "Tun-B detonations (DOE/NV, 2000). During the PA a surface soil sample was collected beneath the drum and analytical results showed no COC were present in the soil (IT, 2002). The drum was screened for radioactivity. After the drum was released it was disposed of at the Area 9 U10c Landfill. All closure activities were documented with photographs and field notes. Clean closure of this site was verified by visual inspection. Verification samples were not required or collected. No further actions are required at this site. 


\subsubsection{CAS 10-22-36: Paint Can}

One empty paint can was located at the site to the south of the crater area from the 1969 "Tun-A through "Tun-D detonations (DOE/NV, 2000). During the PA a surface soil sample was collected beneath the paint can and analytical results showed no COC were present in the soil (IT, 2002). The empty can was removed from the site and disposed of at the Area 9 U10c Landfill. All closure activities were documented with photographs and field notes. Clean closure of this site was verified by visual inspection. Verification samples were not required or collected. No further actions are required at this site.

\subsubsection{CAS 10-22-37: Gas Block}

One partially buried gas block drum was located to the north of the U10ajf crater associated with the 1968 "Tub-C detonation (DOE/NV, 2000). During the PA a surface soil sample was collected adjacent to the east side of the drum and analytical results showed no COC were present in the soil (IT, 2002). The drum was filled with solidified grout and 14 cables of various sizes were protruding from it. Each of the cables was cut and screened for radioactivity. After the drum was released it was disposed of at the Area 9 U10c Landfill. All closure activities were documented with photographs and field notes. Clean closure of this site was verified by visual inspection. Verification samples were not required or collected. No further actions are required at this site.

\subsubsection{CAS 10-24-11: Battery}

One intact automotive battery was removed and sent to recycling. All closure activities were documented with photographs and field notes. Clean closure of this site was verified by visual inspection. Verification samples were not required or collected. No further action is required at this site. 


\subsection{WASTE DISPOSITION}

Wastes generated during the closure of CAU 346: Areas 8, 10 Housekeeping Sites were disposed as follows:

CAS 08-22-11: Several empty drums and buckets were disposed of in the Area 9 U10c Landfill.

CAS 10-14-01: One oil-filled circuit breaker was recycled by the BN Site Utilities Department.

CAS 10-22-06: One grout-filled 208-L (55-gal) gas block drum was disposed of in the Area 9 U10c Landfill.

CAS 10-22-10: One grout-filled 208-L (55-gal) gas block drum was disposed of in the Area 9 U10c Landfill.

CAS 10-22-12: One grout-filled 208-L (55-gal) gas block drum was disposed of in the Area 9 U10c Landfill.

CAS 10-22-13: One grout-filled 208-L (55-gal) gas block drum was disposed of in the Area 9 U10c Landfill.

CAS 10-22-16: One grout-filled 208-L (55-gal) gas block drum was disposed of in the Area 9 U10c Landfill.

CAS 10-22-22: One 151-L (40-gal) drum was disposed of in the Area 9 U10c Landfill.

CAS 10-22-25: One 151-L (40-gal) drum was disposed of in the Area 9 U10c Landfill.

CAS 10-22-36: One empty one-gallon paint can was disposed of in the Area 9 U10c Landfill.

CAS 10-22-37: One grout-filled 208-L (55-gal) drum was disposed of in the Area 9 U10c Landfill.

CAS 10-24-11: One intact automotive battery was recycled by BN Fleet Operations. 
CLOSURE RE PORT - CAU 346

Section: Waste Disposition

Revision : 0

Date: August 2003

THIS PAGE INTENTIONALLY LEFT BLANK 


\subsection{CLOSURE VERIFICATION}

None of the sites required collection of closure verification samples. 
CLOSURE RE PORT - CAU 346

Section: Closure Verification

Revision : 0

Date: August 2003

THIS PAGE INTENTIONALLY LEFT BLANK 


\subsection{SUMMARY AND RECOMMENDATIONS}

\subsection{SUMMARY}

The following site closure activities were performed for the 14 CASs in CAU 346:

All containers (drums, gas block drums, cans, etc.) were disposed of at the Area 9 U10c Landfill.

One intact vehicle battery was removed and sent to BN Fleet Operations for recycling.

One oil-filled circuit breaker was recycled.

\subsection{RECOMMENDATIONS}

Since the closure activities for CAU 346 have been completed following the Nevada Division of Environmental Protection (NDEP)-approved Sectored Clean-up Work Plan for Housekeeping Category Waste Sites (NNSA/NSO, 2003) as documented in this report, NNSA/NSO requests the following:

1. A Notice of Completion be provided by the NDEP to the NNSA/NSO for the closure of all CASs in CAU 346:

CAS 08-22-04

CAS 08-22-11

CAS 08-24-02

CAS 10-14-01

CAS 10-22-06

CAS 10-22-10

CAS 10-22-12

CAS 10-22-13

CAS 10-22-16

CAS $10-22-22$

CAS 10-22-25

CAS 10-22-36

CAS 10-22-37

CAS 10-24-11

2. CAU 346 be moved from Appendix III to Appendix IV of the FFACO Closed Corrective Action Units (FFACO, 1996). 
CLOSURE RE PORT - CAU 346

Section: Summary and Recom.

Revision : 0

Date: August 2003

THIS PAGE INTENTIONALLY LEFT BLANK 


\subsection{REFERENCES}

Bechtel Nevada. 2000. Generic Field Management Plan for Housekeeping Category Waste Sites, Las Vegas, NV.

Bechtel Nevada. 2003. Site-Specific Health and Safety Plan for Corrective Action Units 176 , 346, 350, 351, 352, \& 523 Housekeeping Sites, Nevada Test Site, Nevada, Rev. 1, Las Vegas, NV.

BN, see Bechtel Nevada.

CFR, see Code of Federal Regulations.

Code of Federal Regulations. 2002. Title 40, Part 261.24, “Toxicity Characteristic. Washington D.C.

Code of Federal Regulation. 2002. Title 40, Part 761, "Polychlorinated biphenyls (PCBs) Manufacturing, Processing, Distribution In Commerce, and Use Prohibitions. Washington D.C.

DOE/NV, see U.S. Department of Energy, Nevada Operations Office.

EPA, see U.S. Environmental Protection Agency.

FFACO, see Federal Facility Agreement and Consent Order.

Federal Facility Agreement and Consent Order. 1996 (as amended). Agreed to by the State of Nevada, U.S. Department of Energy, and U.S. Department of Defense.

IT, see IT Corporation.

IT Corporation. 2002. Written communications unpublished data contained in the CAU 346 Project Files "Housekeeping Sampling Report for CAU 346 Areas 8, 10 Housekeeping Sites, Nevada Test Site. Las Vegas, NV.

NAC, see Nevada Administrative Code.

Nevada Administrative Code. 2003. NAC 445A.2272, Contamination of Soil: Establishment of Action Levels. Carson City, NV.

NNSA/NSO, see U.S. Department of Energy, National Nuclear Security Administration Nevada Site Office.

U.S. Department of Energy, National Nuclear Security Administration Nevada Site Office. 2003. Sectored Clean-up Work Plan for Housekeeping Category Waste Sites, Revision 2, DOE/NV--579-REV-2, Las Vegas, NV. 
U.S. Department of Energy, Nevada Operations Office. 2000. United States Nuclear Tests, July 1945 through September 1992. DOE/NV--209 (Rev. 15). Las Vegas, NV.

U.S. Environmental Protection Agency. 1996. Test Methods for Evaluating Solid Waste, Physical/Chemical Methods, EPA Publication SW-846, Third Edition, Washington D.C. 


\section{APPENDIX A}

\section{SAMPLE ANALYTICAL RESULTS}


CLOSURE RE PORT - CAU 346

Section: Appendix A

Revision : 0

Date: August 2003

\section{THIS PAGE INTENTIONALLY LEFT BLANK}




\section{APPENDIX A TABLE OF CONTENTS - SAMPLE ANALYTICAL RESULTS BY SAMPLE DELIVERY GROUP}

Analytical results for the samples collected at the indicated Corrective Action Site (CAS) for Corrective Action Unit 346 are presented in this Appendix. The analytical results are grouped by Sample Delivery Group(s), which are arranged in numerical order in this Appendix. Analytical results for the specific CAS can be found by consulting the indicated Sample Delivery Group(s).

CAS Sample Delivery Group(s)

10-22-22 V1889 and V1890

10-22-25 V1889 and V1890

10-14-01 V1894 
CLOSURE RE PORT - CAU 346 Section: Appendix A

Revision : 0

Date: August 2003

THIS PAGE INTENTIONALLY LEFT BLANK 
CLOSURE RE PORT - CAU 346

Section: Appendix A

Revision : 0

Date: August 2003

\title{
SAMPLE DELIVERY GROUP
}

\author{
V1889
}


CLOSURE RE PORT - CAU 346 Section: Appendix A

Revision : 0

Date: August 2003

THIS PAGE INTENTIONALLY LEFT BLANK 


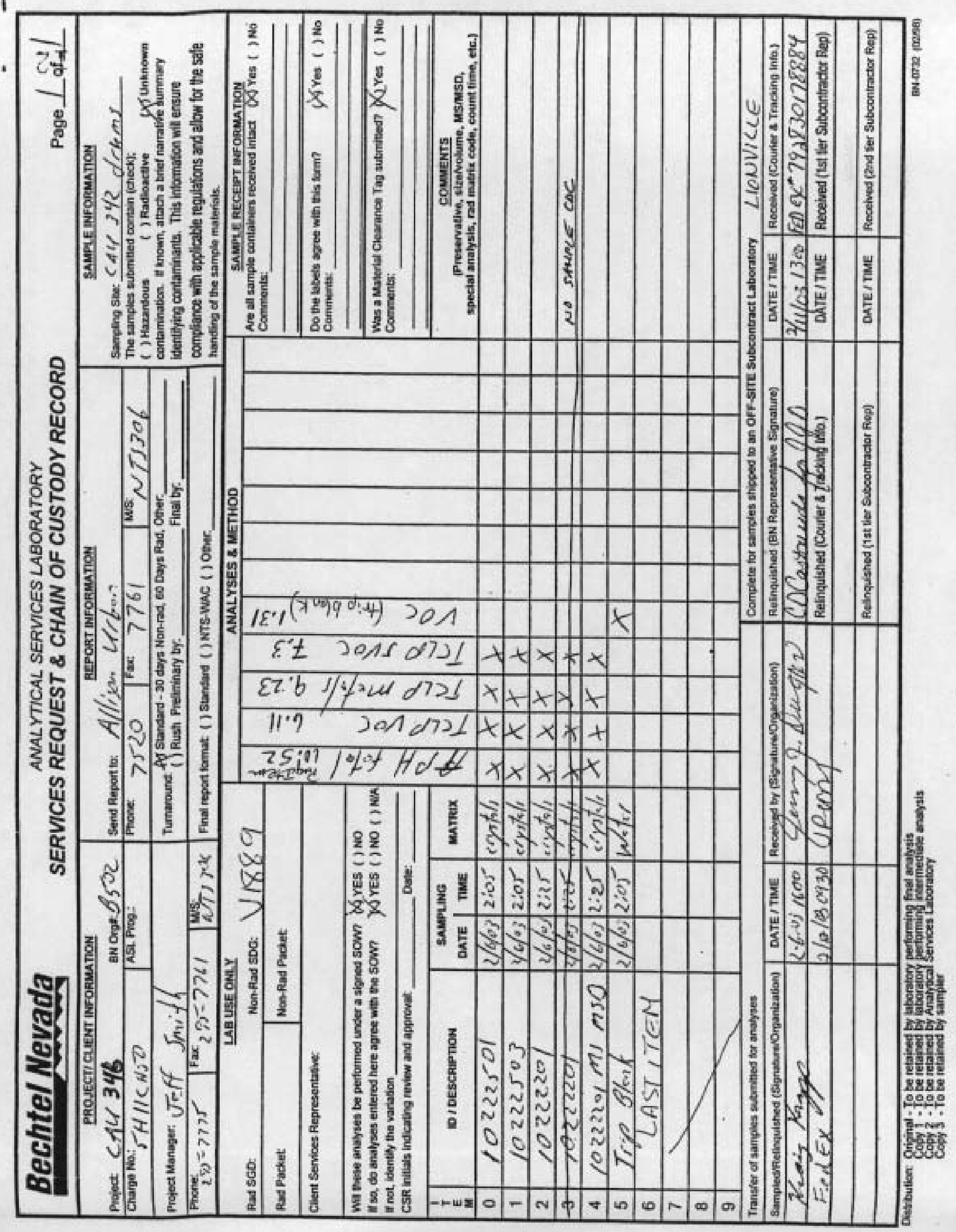


Lionville Laboratory, Inc.

DIBSEL RAMGE ORGMICS BY GC

Report Date: 03/05/03 11:53

RFW Batch Number: 0302 L719 Client: BECHTRL NEVADA Y1889 Work Ordert 60052001001 Page: 1

10222201

\section{Cust ID: 10222501}

10222501

10222503

10222503

10222201

003

Sarmple

Information

$\begin{array}{rr}\text { RFWH: } & 0 \\ \text { Matrix: } & \text { SOLI } \\ \text { D.F.: } & 1\end{array}$

D.F.:

Units:

001

001

1.00

$\mathrm{mg} / \mathrm{kg}$

p-Terpheny 1

$45 \quad t$

SOLID

SOLID

$\mathrm{mg} / \mathrm{kg}$

REPREP

SOLID

48 \%

$\mathrm{mg} / \mathrm{kg}$

Diesel Range Organica

$45=\mathrm{f}$

$28 * 3$

$12.8 \mathrm{U} \quad 12.8 \mathrm{U}$

$12.8 \mathrm{t}$

$\begin{array}{ll}12.8 & 0 \\ & 0\end{array}$

12.8 U

002

SOLID

003

SOLID

1.00

SOLID

Motor Oil

12.8

$12.8 \mathrm{U}$

$\mathrm{mg} / \mathrm{kg}$

33

$\mathrm{mg} / \mathrm{kg}$

1.00

$33 *$ है

17 * *

$\mathrm{mg} / \mathrm{kg}$

RBPREP

$12.8 \quad \mathrm{C}$

$12 \mathrm{~J}$

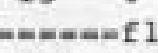

12.8 V

$12.5 \mathrm{v}$

$12.5 \mathrm{U}$

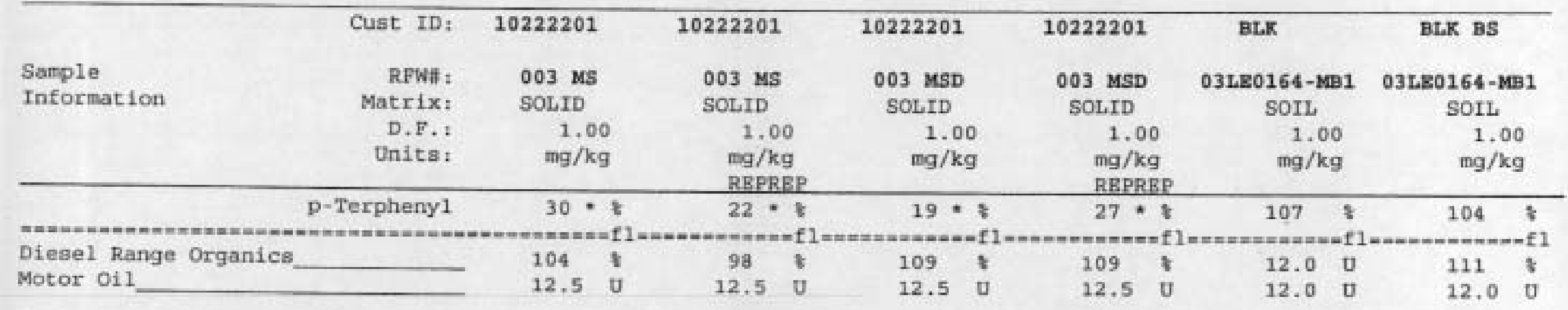

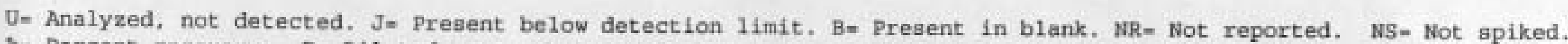

i= Percent recovery. D- Diluted out. Im Interference. NA= Not Applicable. $*=$ Outside of BPA CLP QC

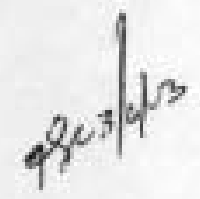




\section{Cust ID: BLK BLK BS}

Sample

Information

$\begin{array}{rcc}\text { RFWH: } & \text { 03LB0209-MB1 } & \text { 03LE0209-MB1 } \\ \text { Matrix: } & \text { SOIL } & \text { SOIL } \\ \text { D.F.: } & 1.00 & 1.00 \\ \text { Units: } & \mathrm{mg} / \mathrm{kg} & \mathrm{mg} / \mathrm{kg}\end{array}$

p-Terphenyl 94 i 92 i

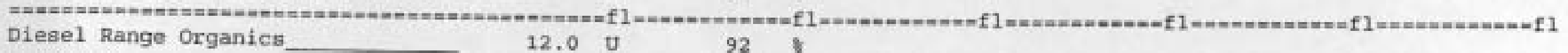

12.0 t

92 을

Motor 011

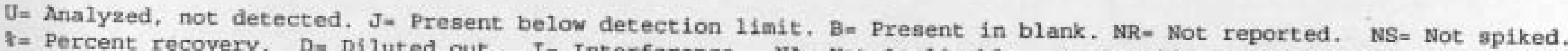
$T=$ Percent recovery. D = Diluted out. Im Interference. NA- Not Applicable. $*=$ Outside of BPA CLP QC 
Lionville Laboratory, Ine.

GAS RANGE ORGANICS

\section{Cust ID:}

10222501

10222501

10222503

10222503

10222201

10222201

Sample

Information

REW\#:

001

001

Matrix: SOLID

D.F.:

1.00

SOLID

Units :

UG/KG

\section{Fluorobenzene}

$0 \times 1$

UG/KG

REPREP

002

SOLID

$29 * *$

1.00
UG/KG

SOLID

ID

UG/KG

$60 \mathrm{~V}$

0 * :

UG/KG

REPREP

Gasoline Range Organics (GRO)

$30 \mathrm{U}$

$36 \mathrm{u}$

$5 * 3$

SOLID

I. 00

1.00
$\mathrm{UG} / \mathrm{KG}$

003

SOLID

DG/KG



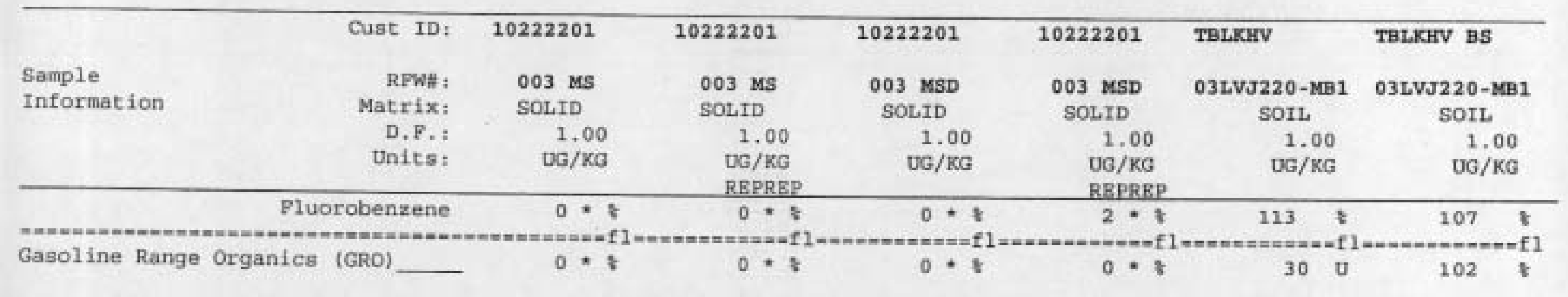

$\mathrm{U}=$ Analyzed, not detected. J- Present below detection limit. B- Present in blank. NR= Not reported. NS- Not spiked.

i= Percent recovery. D = Diluted out. I- Interference, NA= Not Applicable. " Outside of BPA CLP QC 
Lionville Laboratory, Inc.

GAS RANGE ORGANICS

Report Date: 02/26/03 16:16 Client: BECHTEL, NEVADA V1889

Cust ID: TBLKHV BSD

TELKHW

TBLKHW BS

TBLKHW BSD

Sanple

RPWH: 03LVJ220-MB1

03LVJ224-MB1

03LVJ224-MB1

Matrix: SoIL

D.F.: $\quad 1.00$

SOIL

sort

1.00

1.00

03LVJ224-MB1

Dnits:

UG/KG

UG/KG

$\mathrm{DG} / \mathrm{KG}$

SOIL

1.00

UG/KG

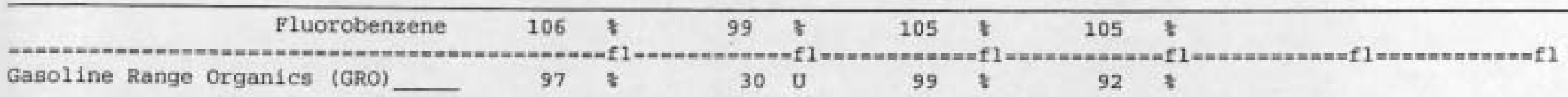

U- Analyzed, not detected. J= Present below detection limit. B- Present in blank. NR= Not reported. NS- Not spiked.

$\mathrm{i}=$ Percent recovery. $\mathrm{D}=$ Diluted out. I= Interference. NA= Not Applicable. * Outside of EPA CLP QC

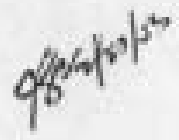


Lionville Laboratory. Inc. Client: BECHTEL NEVADA V1.889 work Order: 60052001001 Page: Ia

\section{Cust ID: TRIP BLANK}

Sample

Information

$\begin{array}{rc}\text { RFWH: } & 004 \\ \text { Matrix: } & \text { WATBR } \\ \text { D.F.: } & 1.00 \\ \text { Units: } & \text { MG/L }\end{array}$

10222501

005
WATER
5.00
MG/L

10222503

D06
WATER
5.00
MG/L

10222201

007
WATBR
5.00
MG/L

VBLKIC

03LVH026-MB1 WATER

$$
1.00
$$$$
\mathrm{MG} / \mathrm{L}
$$

\section{VBLITIB}

03LVX051-MB1 WATBR

$$
1.00
$$

\begin{tabular}{|c|c|c|c|c|c|c|c|c|c|c|c|c|}
\hline Toluene-ds & 101 & 8 & 106 & 8 & 94 & 3 & 99 & 8 & 96 & \% & 98 & है \\
\hline Bromofluorobenzene & 92 & 8 & 107 & ะ & 99 & $\leqslant$ & 100 & 8 & 85 & 5 & 99 & s \\
\hline Recovery 1,2-Dichloroethane-d4 & 92 & 8 & 114 & 3 & 110 & $*$ & 104 & of & 90 & 8 & 102 & 8 \\
\hline \multicolumn{13}{|c|}{ 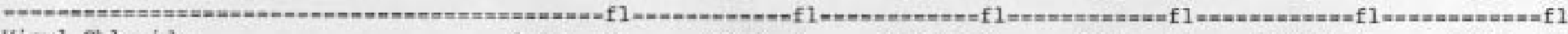 } \\
\hline Vinyl Chloride & 0.010 & $\mathrm{u}$ & 0.050 & 0 & 0.050 & 0 & 0.050 & $\mathrm{U}$ & 0.010 & $\mathrm{U}$ & 0.010 & $\mathrm{U}$ \\
\hline 1,1-Dichloroethene & 0.005 & U & 0.025 & $\mathrm{U}$ & 0.025 & $\mathrm{U}$ & 0.025 & $\mathrm{U}$ & 0.005 & $\mathrm{U}$ & 0.005 & $\mathrm{v}$ \\
\hline Chloroform & 0.005 & u & 0.025 & $\mathrm{U}$ & 0.025 & $\mathrm{U}$ & 0.025 & 0 & 0.005 & $\mathrm{u}$ & 0.005 & v \\
\hline 1.2-Dichloroechane_ & 0.005 & $\mathrm{u}$ & 0.025 & $\mathrm{U}$ & 0.025 & $\mathrm{u}$ & 0.025 & U & 0.005 & $\mathrm{u}$ & 0.005 & $\mathrm{v}$ \\
\hline 2-Butanone & 0.010 & $\mathrm{U}$ & 0.050 & U & 0.050 & $\mathrm{U}$ & 0.050 & 0 & 0.010 & $\mathrm{U}$ & 0.010 & $\mathrm{t}$ \\
\hline Carbon Tetrachloride & 0.005 & u & 0.025 & $\mathrm{U}$ & 0.025 & $\mathrm{u}$ & 0.025 & U & 0.005 & $\mathrm{u}$ & 0.005 & $\mathrm{v}$ \\
\hline Trichloroethene & 0.005 & $\mathrm{U}$ & 0.025 & $\mathrm{u}$ & 0.025 & $\mathrm{u}$ & 0.025 & U & 0.005 & $\mathrm{u}$ & 0.005 & $\mathrm{u}$ \\
\hline Benzene & 0.005 & U & 0.025 & $\mathrm{v}$ & 0.025 & $\mathrm{u}$ & 0.025 & 0 & 0.005 & 0 & 0.005 & D \\
\hline Tetrachloroethene & 0.005 & $\mathrm{u}$ & 0.025 & U & 0.025 & $\mathrm{u}$ & 0.025 & $\mathrm{U}$ & 0.005 & $\mathrm{v}$ & 0.005 & $\mathrm{U}$ \\
\hline Chlorobenzene & 0.005 & $\mathrm{U}$ & 0.025 & $\mathbf{0}$ & 0.025 & $\mathrm{U}$ & 0.025 & U & 0.005 & $\mathrm{u}$ & 0.005 & $\mathrm{D}$ \\
\hline
\end{tabular}

MG/L 
Lionville Laboratory. Inc.

Volatiles by GC/MS, TCLP Leachate

RFW Batch Number: 03022719 Client: BECHTEL NBVADA V1889 Work Order: 60052001001 Page: $2 a$

\section{Cust ID: VBLKIB BS LCHBLK}

Sample

Information

RFWil : 03LVX051-MB1 03LTV009-LB1

Matrix: WATER WATER
D.F.:
1.00
5.00
Units:
$\mathrm{MG} / \mathrm{L}$
$\mathrm{MG} / \mathrm{L}$

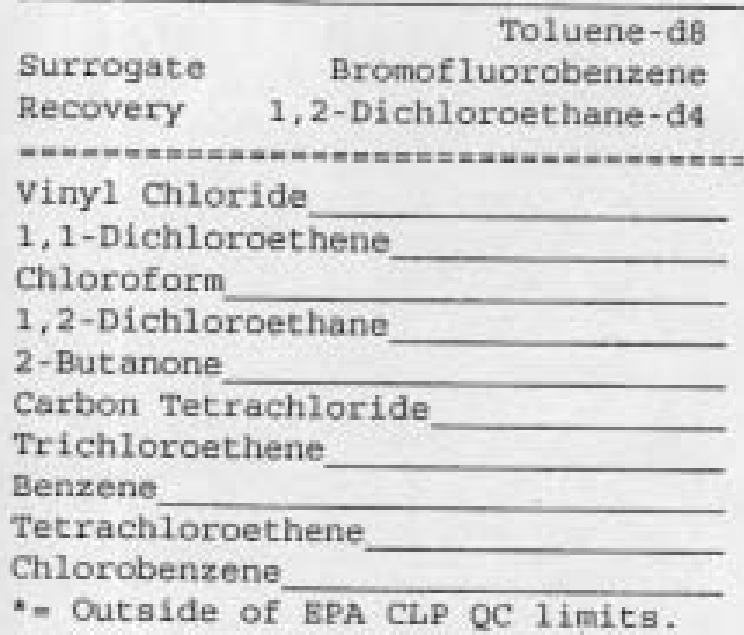

\begin{tabular}{|c|c|c|}
\hline 97 & 8 & 68 \\
\hline 101 & $\xi$ & 91 \\
\hline 111 & 8 & 99 \\
\hline
\end{tabular}

-

111 \% 0.050 प

$110 \div 0.025 \mathrm{t}$

$101 \div 0.025$ 0

$107 \div 0.025$ t

121 \% 0.050 0

$112 \% 0.025$ o

106 के $0.025 \mathrm{U}$

$93 \leqslant 0.025 \mathrm{D}$

$99 \div 0.025 \mathrm{~d}$

$90 \div 0.025$ ए

Report Date: 02/24/03 14 /2

0.025 U

* " Outside of BPA CLP QC limits. 


\section{INORGANIC ANALYSBS DATA SHEET}

Lab Name: LIONVILLE_LABORATORY Lab Code: LVLI Matrix (soil/water): WATBR Level (low/med): * Solids:
Contract: 60052

SAS No.:

LOW

$0 . \overline{0}$
BPA SAMPLE NO.

\section{1}

Lab Sample ID: 0302L719-010

Date Received: 02/12/03

Concentration Units (ug/L or $\mathrm{mg} / \mathrm{kg}$ dry weight): UG/L_

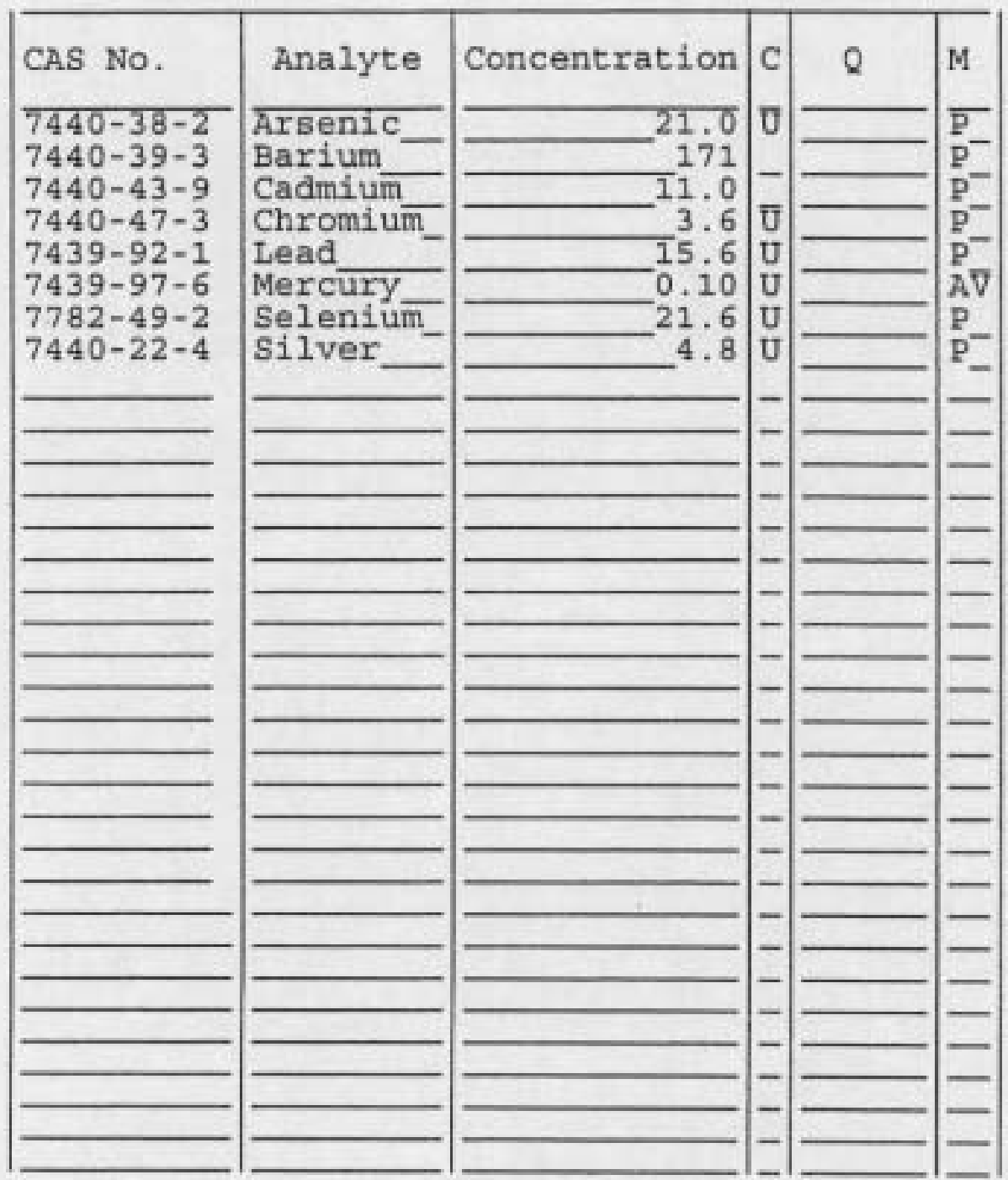

Color Before:

Color After:
Clarity Before:

Clarity After:
Texture:

Artifacts:

\section{Comments :}

10222201

TCLP_OP_O03 
1

INORGANIC ANALYSES DATA SHEET
BPA SAMPLB NO.

\section{1}

Contract: 60052 SAS NO.:

Lab Code: LVLI Matrix (soil/water): WATER Level (low/med): \& Solids:

\section{LOW}

$0 . \overline{0}$
Lab Sample ID: 0302L719-008

Date Received: 02/12/03

Concentration Units (ug/L or $\mathrm{mg} / \mathrm{kg}$ dry weight): UG/L_

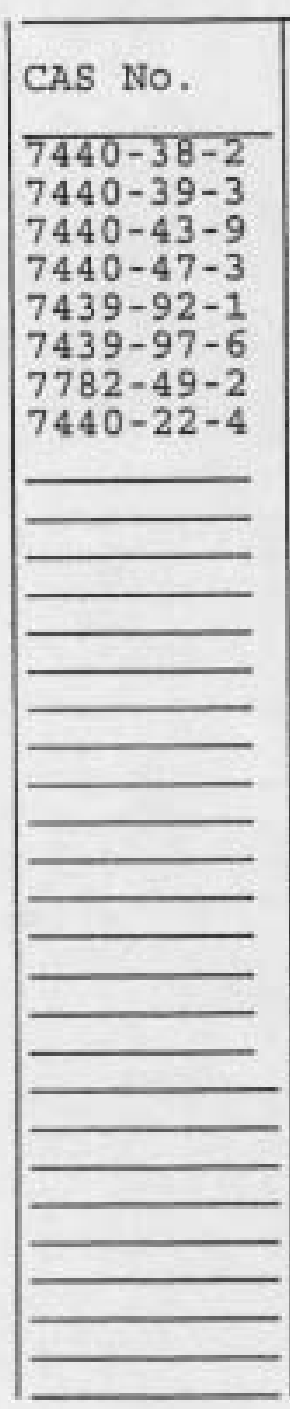

Color Before: Color After:

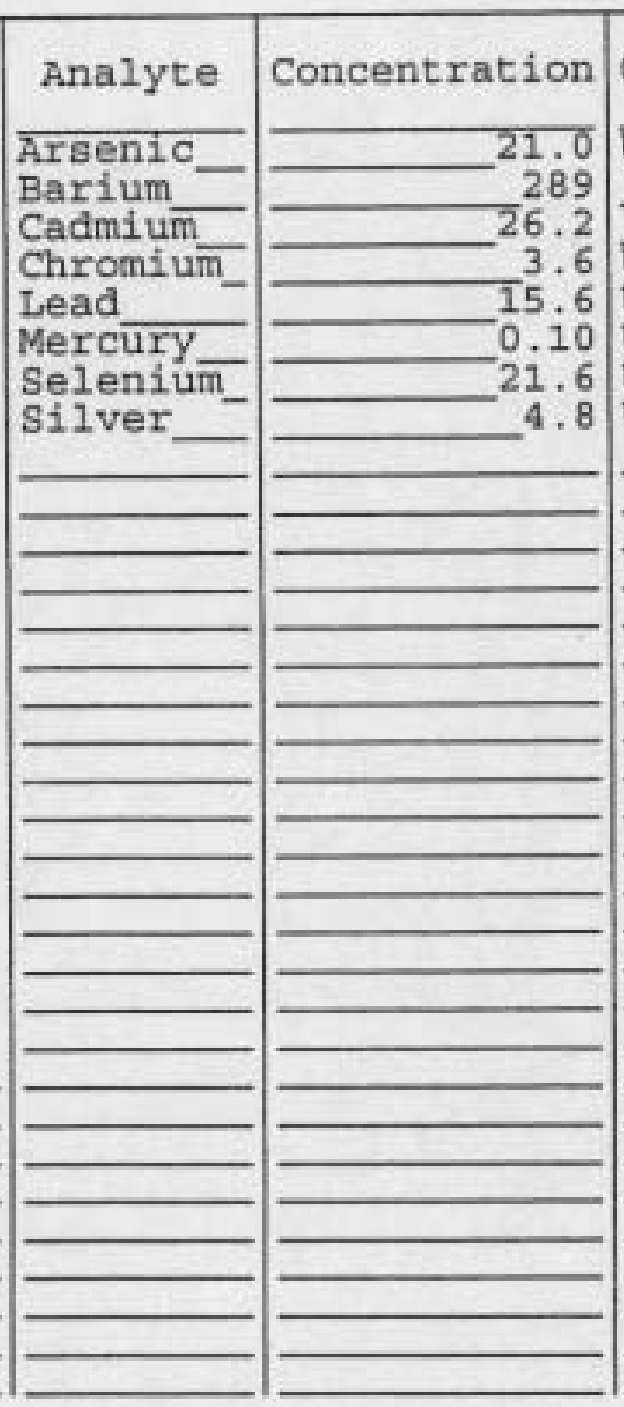

Clarity Before: Clarity After:

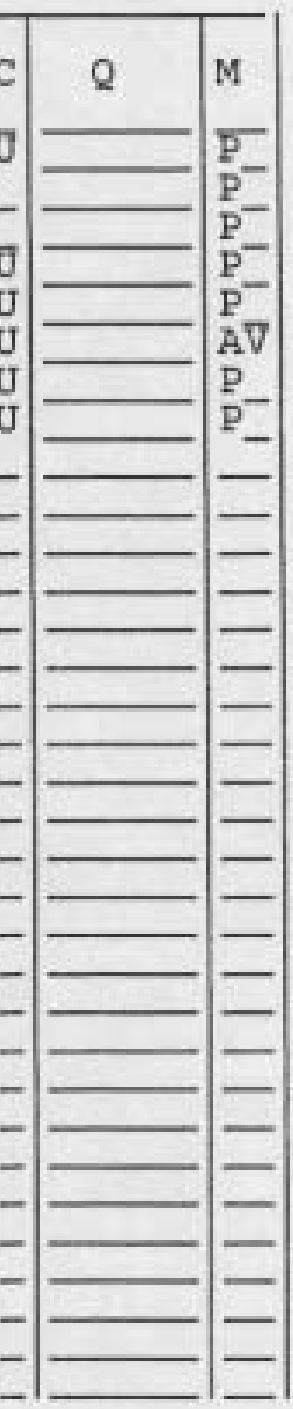

Texture:

Artifacts :

\section{Comments:}

10222501

TCLP_OF_001 
1

INORGANIC ANALYSSS DATA SHBET
EPA SAMPLB NO.

\section{3}

Contract: 60052

SAS No.:

Lab Sample ID: 0302L719-009

Date Received: $02 / 12 / 03$
Lab Name: LION

Matrix (soil/water): WATER

Level (low/med):

\% Solids:
LOW

$0 \overline{0}$

Concentration Units (ug/L or $\mathrm{mg} / \mathrm{kg}$ dry weight): UG/L_

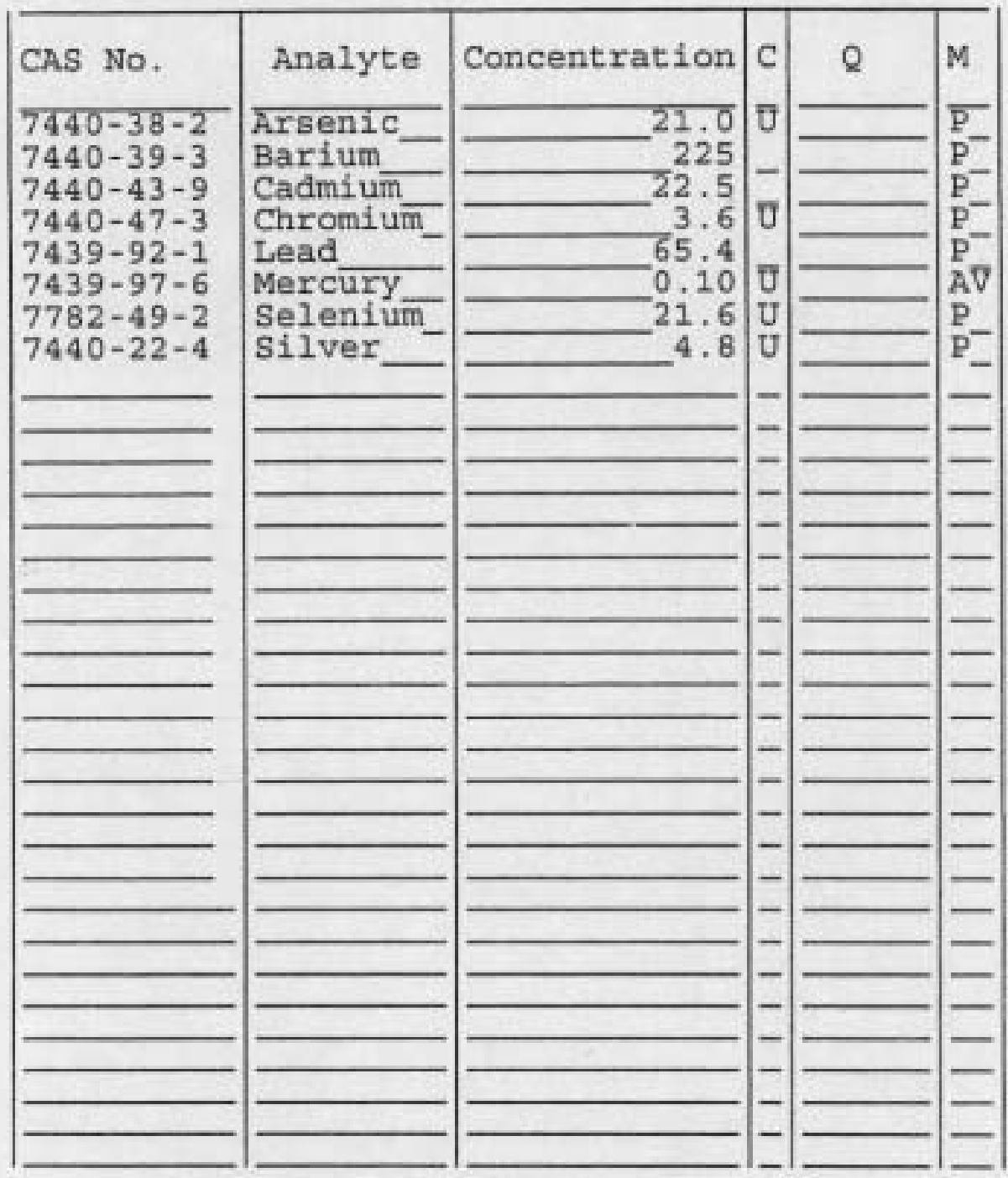

Clarity Before:

Clarity After:
Texture:

Artifacts:

Comments:

10222503

TCLP_OF_OO2 
Lionville Laboratory, Inc.

Semivolatiles by GC/MS, TCLP Leachate Report Date: 02/27/03 12:45

BEW Batch Number: $0302 L 719$ client: BRCHTBL NEVADA V18B9

kork order: 60052001001

Page: la

Cust ID:

10222501

10222501

10222501

10222503

10222201

SBLKar

Sample

Information

$\begin{array}{rc}\text { RFWH: } & \text { O08 } \\ \text { Matrix: } & \text { WATBR } \\ \text { D.F.: } & 1.00 \\ \text { Units: } & \text { MG/L }\end{array}$

$008 \mathrm{MS}$

008 MSD

WATER

1.00
$M G / L$

WATER

$M G / L$

009
WATBR
1.00
MG/L

010
WATBR
1.00
MG/L

03LE0204-MB1

WATER

Nitrobenzene-d5
Surrogate
Recovery $\begin{array}{r}\text { P-Terphenyl-di4 } \\ \text { Phenol-d5 } \\ \text { 2-Pluorophenol }\end{array}$

$\begin{array}{ll}78 & \text { t } \\ 81 & \text { t } \\ 91 & \text { t } \\ 81 & \text { t } \\ 98 & \text { t } \\ 97 & \text { t }\end{array}$

$\begin{array}{rr}75 & \text { t } \\ 76 & t \\ 82 & \text { t } \\ 76 & \text { t } \\ 63 & \text { t } \\ 110 & \text { t }\end{array}$

80
72
96
77
$130+t$
106
$=m=m=m=f l$

Pyridine

1,4-Dichlorobenzene

2-Methylphenol.

3- and/or 4-Methylphenol

Hexachloroethane

Nitrobenzene

Hexachlorobutadiene

2, 4, 6-Trichlorophenol

$\begin{array}{ccc}0.050 \mathrm{D} & 45 \mathrm{~S}\end{array}$

$0.050 \quad \mathrm{v}$

$0.050 \mathrm{U}$

$0.050 \mathrm{U}$

0.050 U

$0.050 \mathrm{U}$

0.050 प

$0.050 \mathrm{U}$

$0.050 \mathrm{U}$

$0.12 \mathrm{U}$

$0.050 \quad \mathrm{U}$

2,4-Dinitrotoluene

Hexachlorobenzene

Pentachlorophenol

$0.050 \quad \mathrm{U}$

$0.12 \mathrm{U}$

$\begin{array}{ll}65 & \text { ? } \\ 85 & \text { ? } \\ 84 & \text { v } \\ 67 & \text { ? } \\ 74 & \text { ? } \\ 67 & \text { ? } \\ 83 & \text { ? } \\ 74 & \text { ? } \\ 74 & \text { ? } \\ 90 & \text { ? } \\ 83 & \text { ? }\end{array}$

$\begin{array}{ll}50 & \text { t } \\ 76 & t \\ 93 & t \\ 93 & t \\ 78 & t \\ 81 & \text { t } \\ 76 & \text { t } \\ 83 & \text { t } \\ 80 & \text { t } \\ 72 & \text { t } \\ 90 & \text { t } \\ 74 & \text { t }\end{array}$

*- Outside of BPA CLP OC Iimits.

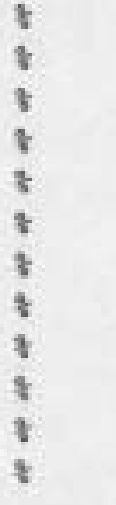

\begin{tabular}{|c|c|}
\hline 89. & $\%$ \\
\hline 80 & * \\
\hline 98 & 3 \\
\hline 81. & $\%$ \\
\hline 104 & $\%$ \\
\hline 96 & t \\
\hline 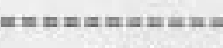 & $=f$ l \\
\hline 0.050 & $\mathrm{U}$ \\
\hline 0.050 & $\mathrm{U}$ \\
\hline 0.050 & $\mathrm{U}$ \\
\hline 0.050 & $\mathrm{U}$ \\
\hline 0.050 & U \\
\hline 0.050 & $\mathrm{U}$ \\
\hline 0.050 & U \\
\hline 0.050 & $\mathrm{U}$ \\
\hline 0.12 & $\mathrm{U}$ \\
\hline 0.050 & $\mathrm{U}$ \\
\hline 0.050 & $\mathrm{U}$ \\
\hline 0.12 & $\mathrm{U}$ \\
\hline
\end{tabular}

\begin{tabular}{rrrr}
87 & है & 80 & है \\
80 & है & 77 & है \\
94 & है & 88 & $\frac{2}{8}$ \\
82 & है & 88 & है \\
109 & है & 101 & है \\
94 & है & 104 & है \\
\hline
\end{tabular}


Lionville Laboratory, Inc.

Cust IDt SBLKNR BS SBLKar BSD LCHBLX

Sample

Information

RFWH: 03LB0204-MB1 03LE0204-MB1 03LTO021-LB1

Matrix: WATER
D.F.:
1.00
$\mathrm{MG} / \mathrm{L}$
1.00
1.00
MG/L
MO/L

WATBR

WATER

Units:

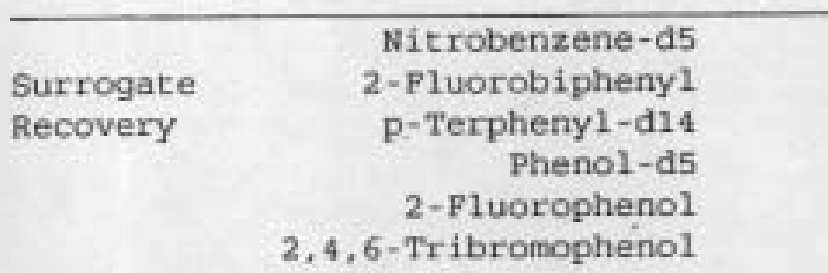

Pyridine

1,4-Dichlorobenzene

2-Methylphenol

3- and/or 4-Methylphenol

Hexachloroethane

Nitrobenzene

Hexachlorobutadiene

$2,4,6$-Trichlorophenol

$2,4,5$-Trichlorophenol

2,4-Dinitrotoluene

Hexachlorobenzene

Pentachlorophenol

\begin{tabular}{|c|c|c|c|c|}
\hline 80 & 1 & 79 & $\frac{1}{8}$ & 91 \\
\hline 73 & 1 & 74 & 3 & 85 \\
\hline 94 & t & 97 & $y$ & 90 \\
\hline 77 & $t$ & 77 & \% & 89 \\
\hline 97 & 8 & 95 & 7 & 111 \\
\hline 97 & \& & 105 & 8 & 105 \\
\hline
\end{tabular}

- Outside of BPA CLP QC 1imits. 
CLOSURE RE PORT - CAU 346

Section: Appendix A

Revision : 0

Date: August 2003

\section{SAMPLE DELIVERY GROUP}

V1890 
CLOSURE RE PORT - CAU 346

Section: Appendix A

Revision : 0

Date: August 2003

\section{THIS PAGE INTENTIONALLY LEFT BLANK}




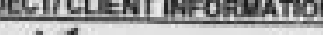

\begin{tabular}{|c|c|c|c|c|c|}
\hline Propect $C 4434$ & 6 & 0502 & Send Report tax & $4 / 6 \mathrm{en}$ & \\
\hline Charge No: $5 \mathrm{H} / / \mathrm{C}$ & 450 & कि: & Phone: $255-7520$ & क्रि, $55-7761$ & WE: $\sqrt{71306}$ \\
\hline Project Marager $J_{e f}$ & $f \sqrt{n} \cdot i t h$ & & 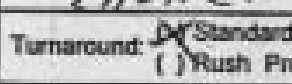 & $\begin{array}{l}0 \text { ders Non-rad } 60 \text { Days Fa } \\
\text { inary by: }\end{array}$ & Final ty. \\
\hline Phone $27,=7775$ & axc $275=7761$ & W55 306 & Final report format: () st & tard () NTS-W & 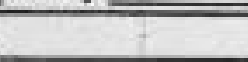 \\
\hline
\end{tabular}

\section{Sanpling sien caU $34 / 2$ drum)} The samples sermitied contan (check: Do Untanown (1) Haxarsous () Radbasctive f Junknown contamination. Whown, atach a brief norntive bummary identlying combeninamts. This inforrabion will ensure handiling of the sample materials.

\begin{tabular}{|c|c|}
\hline \multicolumn{2}{|c|}{ LAB USE ONLY } \\
\hline Rad SGD. & Non-Rad soc: \\
\hline Plad Packet & Non-Pad Pachut \\
\hline
\end{tabular}

Clent Senvices Representative:

Wir these andyses be pertomed under a signed sow? (M/f's () NO

If sa, do analyses enlered here agres with the sown. (U/YES ( ) NO ( ) NWE

If not, identily the variation

CSR initals insicaling review and appreval:

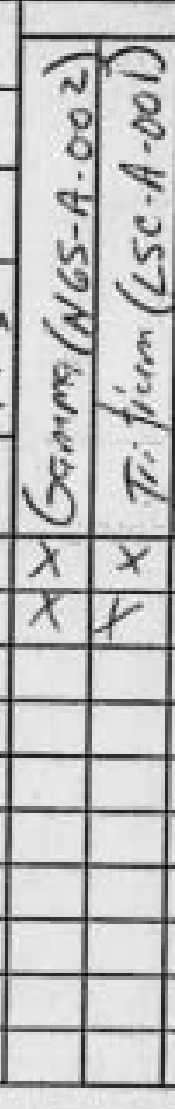

ANALYSES \& METHOD

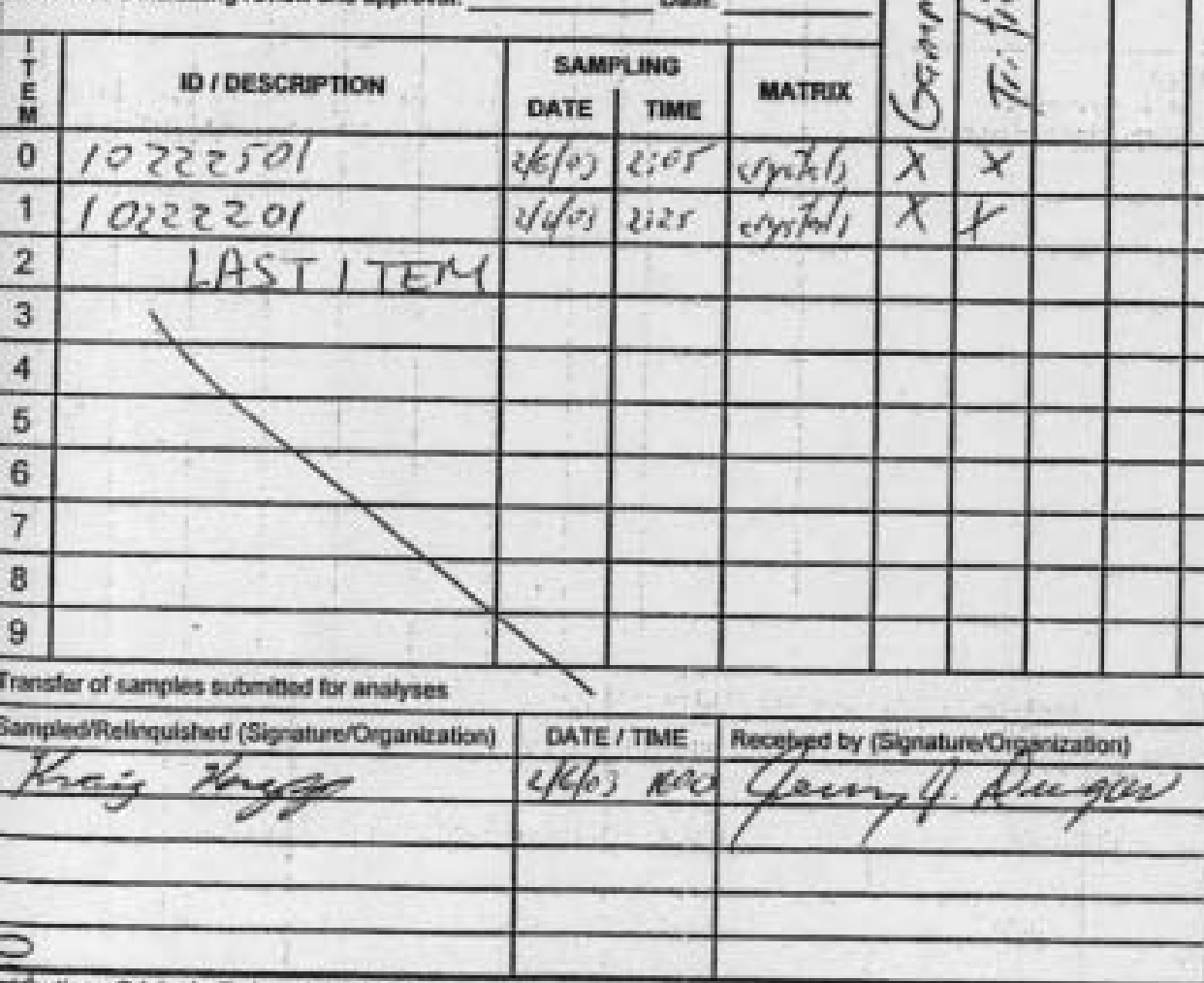

NiTSOB- 4005

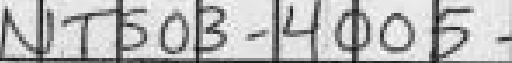

II. special analysis, rad matrtx code, count time, etc: Comments:

Do the labels agree with this farm?

(ufres i ) No

Comments:

Was a Malertal Clearance Tag submitied? MY Yes ( ) No Commenta:

Complete for sarnples shipped to an OFF-sire subcontract Laboratory $5 \mathrm{C} \times \mathrm{A}$

\begin{tabular}{|l|l|l|l} 
Relinquished (BN Representabive Signature) & DATE/ TME & Recelved (Courler \& Tracking Info)
\end{tabular}

CQactounde hala

Relinquished (Courier 8 thackitginto.)

791294250470

Relloquished (1st ber Subcontractor Rep)

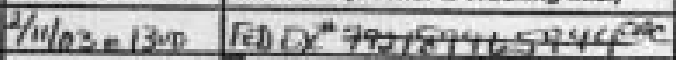
DATE $/$ TIME Tfiecelved (fst lier Subcontoctor Rep) $42630930 \times 791294250470$ DATE/TME Received (2nd ber Sybconbactor Rep)

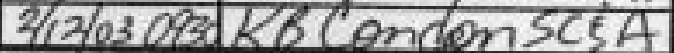




\section{CASE NARRATIVE \\ SDG V1810 \\ Laboratory Report Identification Number: 4005}

February 26, 2003

\section{Introduction}

On February 12, 2003, two soil samples, (SDG V1890), were received for analysis at the Sanford Cohen and Associates (SC\&A) Southeastern Environmental Laboratory, located in Montgomery, Alabama. The chain-of-custody accompanying the samples requested that it be analyzed within 45 days of receipt at the laboratory. The turn-around-time was requested to be changed to 14 days by e-mail on February 12, 2003. The samples were analyzed in accordance with the Bechtel Nevada Services Subcontract Task Order Agreement Form, Exhibit B, Statement of Work and Specifications, Rev 1, 1/23/01.

\section{Analytical Methodology}

The radioanalytical results reported for each sample include the site and laboratory sample identification numbers, collection date, method of analysis, and the quality control samples that were analyzed concurrently. Samples were analyzed in accordance with the following method.

\begin{tabular}{||l|l|l|l||}
\hline \multicolumn{1}{|c|}{ Radionuclide } & \multicolumn{1}{|c|}{$\begin{array}{c}\text { Method } \\
\text { Number }\end{array}$} & \multicolumn{1}{|c|}{$\begin{array}{c}\text { Method } \\
\text { Name }\end{array}$} & \multicolumn{1}{|c|}{$\begin{array}{c}\text { Counting } \\
\text { Method }\end{array}$} \\
\hline Tritium & EPA 906.0 & Tritium & $\begin{array}{l}\text { Liquid } \\
\text { Scintillation }\end{array}$ \\
\hline $\begin{array}{l}\text { Gamma Emitting } \\
\text { Radionuclides }\end{array}$ & EPA 901.1 & $\begin{array}{l}\text { Gamma Emitting } \\
\text { Radionuclides }\end{array}$ & $\begin{array}{l}\text { Gamma } \\
\text { Spectrometry }\end{array}$ \\
\hline \hline
\end{tabular}

\section{Analytical Results}

\section{Deficiencies}

None.

\section{Matrix Interferences}

There were no indications of matrix interference. 


\section{Dilutions}

There were no dilutions.

\section{Detection Limits}

The required detection limits (RDL) were met for all analyses.

\section{Reanalysis}

There were no reanalysis.

Deviations from Protocols

There were no deviations from the written protocols and analytical methods.

\section{Contacts with the CTR}

There was no contact with the CTR regarding these samples.

\section{Quality Control}

Site Samples Used for Quality Control Samples:

\begin{tabular}{|c|c|c|}
\hline Site Sample Number & Laboratory Sample Number & $\begin{array}{c}\text { Type of Quality Control Analysis } \\
\text { Sample }\end{array}$ \\
\hline \hline Laboratory Type II Water & SCAQC-4005-LCI & Laboratory Control Sample \\
\hline 10222501 & SCAQC-4005-LDI & Laboratory Duplicate Sample \\
\hline Laboratory Type II Water & SCAQC4005-PB & Preparation Blank \\
\hline
\end{tabular}

The analytical results of all quality control samples met the acceptance criteria specified in the sow.

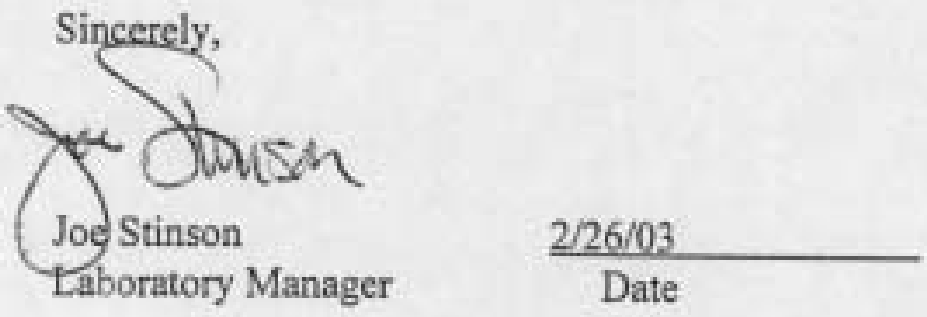


Sample and QC Sample Results Summary 


\section{Sanford Cohen \& Associates \\ Southeastern Environmental Laboratory}

\section{Radioanalytical Results}

Report Identification Number: V18s0

\begin{tabular}{|c|c|c|c|c|c|c|}
\hline $\begin{array}{l}\text { Project Name: } \\
\text { Site Sample ID: } \\
\text { Other Sample ID: }\end{array}$ & $\begin{array}{l}\text { Bechtel Neveda } \\
10222501\end{array}$ & Chain-of-Cu & $\begin{array}{l}\text { on Date: } 2 \\
\text { Number: } 4\end{array}$ & $05: 00 \mathrm{AM}$ & $\begin{array}{l}\text { Date Received: } \\
\text { Laboratory Code: }\end{array}$ & $\frac{2 / 12 / 2000}{S C A}$ \\
\hline Mathod Number & Badionuclide & $\begin{array}{l}\text { Laboratory } \\
\text { Sample ID }\end{array}$ & $\begin{array}{l}\text { Activity } \\
\text { incila) }\end{array}$ & $\begin{array}{l}2 \text { a TPU } \\
(\mathrm{pCla})\end{array}$ & $\begin{array}{l}\text { Total Error } \\
\text { (nclial } \\
\end{array}$ & $\begin{array}{l}\text { MDA } \\
\text { (DCiVe) }\end{array}$ \\
\hline EPA 901.1 & $K-40$ & NTS03-4005-01 & 1.21 & 0.230 & 0.260 & 0.158 \\
\hline EPA 901.1 & $\mathrm{CO}-60$ & NTS03-4005-01 & 0.004 & 0.010 & 0.010 & 0.017 \\
\hline EPA 901.1 & $Y-88$ & NTS03-4005-01 & -0.003 & 0.009 & 0.009 & 0.016 \\
\hline EPA 901.1 & RU-106 & NTS03-4005-01 & -0.000 & 0.078 & 0.078 & 0.132 \\
\hline EPA 901.1 & SB-125 & NTS03-4005-01 & -0.003 & 0.023 & 0.023 & 0.099 \\
\hline EPA 901.1 & Cs-134 & NTS03-4005-01 & -0.002 & 0.010 & 0.010 & 0.014 \\
\hline EPA 901.1 & CS-137 & NTS03-4005-01 & 0.017 & 0.009 & 0.010 & 0.017 \\
\hline EPA 901.1 & CE-144 & NTS03-4005-01 & -0.153 & 0,499 & 0.500 & 0.088 \\
\hline EPA 901.1 & PM-144 & NTS03-4005-01 & 0.003 & 0.008 & 0.008 & 0.013 \\
\hline EPA 901.1 & PM-146 & NTS03-4005-01 & 0.010 & 0.011 & 0.011 & 0.019 \\
\hline EPA 901.1 & EU-152 & NTS03-4005-01 & 0.007 & 0.018 & 0.018 & 0.032 \\
\hline EPA 801.1 & EU-154 & NTS03-4005-01 & 0.000 & 0.000 & 0.000 & 0.022 \\
\hline EPA 901,1 & EU-155 & NTS03-4005-01 & 0.003 & 0.022 & 0.022 & 0.039 \\
\hline EPA 901.1 & $\mathrm{~PB}-212$ & NTS03-4005-01 & 0.018 & 0.019 & 0.019 & 0.027 \\
\hline EPA 901.1 & AC-228 & NTS03-4005-01 & 0.001 & 0.036 & 0.036 & 0.063 \\
\hline EPA 901.1 & TH.234 & NTS03-4005-01 & 0.053 & 0.165 & 0,165 & 0.284 \\
\hline EPA 901.1 & U-235 & NTSC3-4005-01 & 0.001 & 0.014 & 0.014 & 0.022 \\
\hline EPA 901.1 & U.238 & NTS03-4005-01 & 0.506 & 1.86 & 1.86 & 3.03 \\
\hline EPA 901.1 & AM-241 & NTS03-4005-01 & -0.011 & 0.044 & 0.044 & 0.077 \\
\hline EPA 906.0 & $\mathrm{H}-3$ & NTS03-4005-01 & 0.005 & 0.008 & 0.008 & 0.014 \\
\hline
\end{tabular}

\begin{tabular}{|c|c|c|c|c|}
\hline \multicolumn{5}{|c|}{ Quality Control Samples } \\
\hline Radionuclida & Laborelory Contro: (LC) & Laborater Duplicate [LD) & Matrix Spike (MS) & Preseration Blank (PB) \\
\hline Gamma & SCAQC-4005-LC1 & SCAQC-4005-LD1 & & SCAQC-4005-PB \\
\hline Tritium & SCAQC-4005-LC1 & SCAOC-4005-LD1 & SCAQC-4005-MS1 & SCAQC-4005-PB \\
\hline
\end{tabular}




\section{Sanford Cohen \& Associates \\ Southeastern Environmental Laboratory}

Radioanalytical Results

Report Identification Number: V1890

\begin{tabular}{|c|c|c|c|c|c|c|}
\hline \multicolumn{2}{|c|}{$\begin{array}{l}\text { Project Name: Bechtel Neveda } \\
\text { Ste Sample ID: } 10222201 \\
\text { Other Sample ID: }\end{array}$} & \multicolumn{3}{|c|}{ Chain-of-Custody Number. NONE } & \multicolumn{2}{|c|}{ Matrix Sol } \\
\hline Methed Number & Badianuclide & $\begin{array}{l}\text { Laboratory } \\
\text { Sample 10 } \\
\end{array}$ & $\begin{array}{l}\text { Activity } \\
\text { (oCilp) }\end{array}$ & $\begin{array}{l}2 \text { o TPU } \\
(0 \mathrm{CH} / \mathrm{g})\end{array}$ & $\begin{array}{c}\text { Total Errer } \\
(0 \mathrm{C} \text { (b) } \\
\end{array}$ & $\begin{array}{l}\text { MDA } \\
\text { (oCila) }\end{array}$ \\
\hline EPA 901.1 & $\mathrm{~K}-40$ & NTS0S-4005-02 & 4.17 & 0.564 & 0.702 & 0.210 \\
\hline EPA 901.1 & $00-60$ & NTS03-4005-02 & 0.009 & 0.013 & 0.013 & 0.023 \\
\hline EPA 901.1 & $Y-88$ & NTS03-4005-02 & 0.003 & 0.012 & 0.012 & 0.023 \\
\hline EPA 901.1 & $R U-106$ & NTS03-4005-02 & 0.004 & 0.102 & 0.102 & 0.182 \\
\hline EPA 901.1 & SB-125 & NTSO3-4005-02 & -0.006 & 0.031 & 0.031 & 0.052 \\
\hline EPA 901.1 & $\operatorname{CS}-134$ & NTS03-4005-02 & $-0,002$ & 0.012 & 0.012 & 0.019 \\
\hline EPA 901.1 & CS-137 & NTS03-4005-02 & 0.051 & 0.020 & 0.021 & 0.020 \\
\hline EPA 901.1 & CE-144 & NTSO3-4005-02 & -1.22 & 0.666 & 0.677 & 0.125 \\
\hline EPA 901.1 & PM-144 & NTS03-4005- 62 & 0.001 & 0.011 & 0.011 & 0.017 \\
\hline EPA 901,1 & $P M-146$ & NTS03-4005-02 & 0.007 & 0.015 & 0.015 & 0.027 \\
\hline EPA 901.1 & EU-152 & NTS03-4005-02 & -0.012 & 0.026 & 0.026 & 0.044 \\
\hline EPA 901.1 & EU-154 & NTS03-4005-02 & 0.000 & 0.000 & 0.000 & 0.031 \\
\hline EPA 901.1 & EU-155 & NTS03-4005-02 & 0.042 & 0.030 & 0.030 & 0.053 \\
\hline EPA 901.1 & PB-212 & NTS03-4005-02 & 0.019 & 0.026 & 0.025 & 0.040 \\
\hline EPA S01.1 & AC-228 & NTS03-4005-02 & -0.012 & 0.050 & 0.050 & 0.083 \\
\hline EPA 901.1 & TH-234 & NTSO3-4006.02 & 0.053 & 0.203 & 0.203 & 0.345 \\
\hline EPA 901.1 & U-235 & NTS03-4005-02 & $-0,023$ & 0.020 & 0.020 & 0.030 \\
\hline EPA 901.1 & U.238 & NTS03-4005-02 & 1.05 & 2.48 & 2.48 & 4.09 \\
\hline EPA 901.1 & AM-241 & NTS03-4005-02 & 0.022 & 0.047 & 0.048 & 0.084 \\
\hline EPA 906.0 & $\mathrm{H}-3$ & NTSO3-4005-02 & 0.021 & 0,014 & 0.014 & 0.021 \\
\hline
\end{tabular}

\begin{tabular}{|c|c|c|c|c|}
\hline \multirow[b]{2}{*}{ Badienuclide } & \multirow[b]{2}{*}{ Laberatery Control (LC) } & \multicolumn{2}{|c|}{ Quality Control Samplos } & \multirow[b]{2}{*}{ Presaration Blank (PB) } \\
\hline & & Laberatory Duplicate (L.DA & Matrix Splke ous] & \\
\hline Gamma & SCAQC-4005-LC1 & SCAQC-4005-LD1 & & $S C A C C-4005-P B$ \\
\hline Tritium & SCAQC-4005-LC1 & SCAQC-4005-LD1 & SCAQC-40OS-MS1 & SCAOC-4005-PB \\
\hline
\end{tabular}




\section{Sanford Cohen \& Associates \\ Southeastern Environmental Laboratory}

Radioanalytical Results

Quality Control Sample

Laboratory Control (LC1)

Report Identification Number V1890

\begin{tabular}{|c|c|c|c|}
\hline $\begin{array}{l}\text { Project Name: Bechtel Nevada } \\
\text { site Sample ID: N/A }\end{array}$ & Chain-of-Custody Number: None & Matrix: & Soil \\
\hline Other Sample ID: LC1 & Collection Date: 2/12/2003 13000 AM & $\begin{array}{l}\text { Date Received: } \\
\text { Laboratory Code: }\end{array}$ & $\frac{2 / 12 / 2003}{\text { SCA }}$ \\
\hline
\end{tabular}

\begin{tabular}{|c|c|c|c|c|c|c|}
\hline Method Number & Radionuclide & $\begin{array}{l}\text { Laboratory } \\
\text { Sample ID } \\
\end{array}$ & $\begin{array}{l}\text { Activity } \\
\text { (pcila) }\end{array}$ & $\begin{array}{l}2 \text { a TPU } \\
(0 \mathrm{CV}(\mathrm{g})\end{array}$ & $\begin{array}{l}\text { Total Error } \\
\text { (DCly) } \\
\end{array}$ & $\begin{array}{l}\text { MDA } \\
\text { (ocila) }\end{array}$ \\
\hline EPA 901.1 & $\mathrm{CO}-60$ & SCAQC-4005-LC1 & 17.9 & 1.65 & 2.43 & 0.079 \\
\hline EPA 901.1 & CD-109 & SCAQC-4005-LC1 & 14.0 & 3.08 & 3.38 & 2.31 \\
\hline EPA 901.1 & $\operatorname{cs}-137$ & SCAQC-4005-LC1 & 25.0 & 2.65 & 3.64 & 0.124 \\
\hline EPA 906.0 & $\mathrm{H} \cdot 3$ & SCAQC-4005-LC1 & 2000 & 311 & 520 & 354 \\
\hline
\end{tabular}

\begin{tabular}{|lllll|}
\hline Radianuctide & \multicolumn{2}{c|}{ Quality Control Samples } \\
\hline Tritium & SCAOC-4005-LC1 & SCAQC-4005-LD1 & SCACC-4005-MS1 & Preparation Blank.PB) \\
Gamma & SCAOC-4005-LC1 & SCAOC-4005-LD1 & & SCACC-4005-PB \\
\hline
\end{tabular}




\section{Sanford Cohen \& Associates \\ Southeastern Environmental Laboratory}

Radioanalytical Results

\section{Quality Control Sample \\ Duplieate (LD1)}

Report Identification Number: Vis9o

\begin{tabular}{|c|c|c|c|}
\hline $\begin{array}{l}\text { Project Name: Bechtel Nevada } \\
\text { Ste Sample ID: 10222801 }\end{array}$ & Chain-of-Custody Number, NONF & Matric & Soil \\
\hline Other Sample ID: LDI & Collection Date: 2/5/20032.05:00 AM & $\begin{array}{l}\text { Date Received: } \\
\text { Laboratory Code: }\end{array}$ & $\begin{array}{l}2 / 12 / 2003 \\
\text { sCA }\end{array}$ \\
\hline
\end{tabular}

\begin{tabular}{|c|c|c|c|c|c|c|}
\hline Mechod Number & Badionuclide & $\begin{array}{l}\text { Laboratory } \\
\text { Sampla } 10 \\
\end{array}$ & $\begin{array}{l}\text { Activily } \\
\text { (nCivi) }\end{array}$ & $\begin{array}{l}2 \text { a TPU } \\
\text { (pCih) }\end{array}$ & $\begin{array}{c}\text { Total Errer } \\
\text { (nCla) } \\
\end{array}$ & $\begin{array}{l}\text { MDA } \\
(0 C \mathrm{C}(a)\end{array}$ \\
\hline EPA 901.1 & $K-40$ & SCAOC-4005-LD1 & 1.07 & 0.250 & 0.272 & 0.178 \\
\hline EPA 901.1 & $00-60$ & SCACC-4005-LD1 & -0.001 & 0.010 & 0.010 & 0.016 \\
\hline EPA 901.1 & $Y-88$ & SCAOC-4005-LD1 & -0.005 & 0.009 & 0.009 & 0.015 \\
\hline EPA 901.1 & $R U-106$ & SCAQC-4005-LD1 & 0.063 & 0.081 & 0.082 & 0.145 \\
\hline EPA 901.1 & $58-125$ & SCAQC-4005-LD1 & -0.003 & 0.022 & 0.022 & 0.038 \\
\hline EPA 901.1 & CS-134 & SCAQC-4005-LD1 & 0.006 & 0.010 & 0.010 & 0.016 \\
\hline EPA 901,1 & CS-137 & SCAQC-4005-LD1 & -0.004 & 0.010 & 0.010 & 0.016 \\
\hline EPA 901.1 & CE-144 & SCAQC-4005-LD1 & -0.171 & 0.546 & 0.546 & 0.086 \\
\hline EPA 901.1 & PM-144 & SCAQC-4005-LD1 & 0.002 & 0.009 & 0.008 & 0.013 \\
\hline EPA 901.1 & PM-146 & SCAQC-400S-LD1 & -0.007 & 0.011 & 0.011 & 0.018 \\
\hline EPA 901.1 & EU-152 & SCAQC-4005-LD1 & 0.003 & 0.018 & 0.018 & 0.032 \\
\hline EPA 901.1 & EU-154 & SCAOC-4005-LD1 & 0.000 & 0.000 & 0.000 & 0.022 \\
\hline EPA 901.1 & EU-155 & SCAQC-4005-LD1 & 0.002 & 0.023 & 0.023 & 0.040 \\
\hline EPA 901.1 & PB-212 & SCAOC-4005-LD1 & -0.000 & 0.017 & 0.017 & 0.026 \\
\hline EPA 901.1 & $A C-228$ & SCAQC-4005-LD1 & -0.015 & 0.035 & 0.035 & 0.060 \\
\hline EPA 901.1 & $\mathrm{TH}-234$ & SCAQC-40C5-LD1 & 0.144 & 0.165 & 0.166 & 0.286 \\
\hline EPA 901.1 & U.235 & SCAQC-4005-LD1 & 0.004 & 0.014 & 0.014 & 0.023 \\
\hline EPA 901.1 & $4-238$ & SCAQC-4005-LDI & -1.22 & 1.79 & 1.80 & 2.65 \\
\hline EPA 901.1 & AM-241 & SCAOC-4005-LDI & -0.026 & 0.044 & 0.044 & 0.076 \\
\hline EPA 906.0 & $\mathrm{H}-3$ & SCAQC-4005-LD1 & 0.006 & 0.008 & 0.009 & 0.014 \\
\hline
\end{tabular}

\begin{tabular}{|c|c|c|}
\hline \multicolumn{3}{|c|}{ Laboratory Samples for Duplicates } \\
\hline Reslienuclide & $\begin{array}{l}\text { Laboratory } \\
\text { Semple ID }\end{array}$ & $\begin{array}{l}\text { Duplieate of } \\
\text { Semole iD }\end{array}$ \\
\hline GAMMA & SCAQC-4005-LD1 & NTS03-4006-01 \\
\hline $\mathrm{H} \cdot 3$ & SCAQC-4005-LD1 & NTS03-4005-01 \\
\hline
\end{tabular}

\begin{tabular}{|c|c|c|c|c|}
\hline \multicolumn{5}{|c|}{ Quality Control Samples } \\
\hline Radienuelide & Laboratory Control(RC) & Laborntory Duplicate (LD) & Natrix Spike (MSI & Preparation Blank (PG) \\
\hline Trthlum & SCACC-4005-LC1 & SCACC-4005-LD1 & SCACC-4005-MS1 & $S C A C C-4005-P B$ \\
\hline Gamma & SCAQC-4005-LC1 & SCAQC-4005-LDI & & SCAQC-400S-PB \\
\hline
\end{tabular}




\section{Sanford Cohen \& Associates \\ Southeastern Environmental Laboratory}

Radioanalytical Results

Quality Control Sample

Matrix Splke (MS1)

Report Identification Number: V1890

Project Name: Bechtel Newada

Site Sample ID: 10222501

Cener Sample ID: MSI
Chain-of-Custody Number, Nane

Collection Date: 2/6/2003 2:05:00 AM
Matroc Soil

Date Received: 2/12/2003

Laboratory Code: SCA

\begin{tabular}{|c|c|c|c|c|c|c|}
\hline Method Number & Badionuclide & $\begin{array}{l}\text { Labcratory } \\
\text { Sample iD } \\
\end{array}$ & $\begin{array}{l}\text { Activity } \\
\text { (ncila) }\end{array}$ & $\begin{array}{l}2 \text { a TPU } \\
(0 \mathrm{CH}(\mathrm{d})\end{array}$ & $\begin{array}{c}\text { Total Error } \\
\text { (oCilg) } \\
\end{array}$ & $\begin{array}{l}\text { MOA } \\
(\mathrm{nCS}(\mathrm{a})\end{array}$ \\
\hline EPA 906.0 & $\mathrm{H} \cdot 3$ & SCAOC-4005-MS1 & 9.50 & 0.111 & 1.90 & 0.017 \\
\hline
\end{tabular}

\begin{tabular}{|c|c|c|c|c|}
\hline \multicolumn{5}{|c|}{ Quality Control Samples } \\
\hline Bartionuclide & Laboratery CentroL(LC) & Laberatory Duplicate (LD) & Matrix Spike (MS) & Preparation Blark (PB) \\
\hline Tritium & SCAOC-4005-LC1 & SCAQC-4005-LD1 & SCAOC-4005-MS1 & SCAQC $4005-P B$ \\
\hline
\end{tabular}


CLOSURE RE PORT - CAU 346 Section: Appendix A

Revision : 0

Date: August 2003

THIS PAGE INTENTIONALLY LEFT BLANK 
CLOSURE RE PORT - CAU 346

Section: Appendix A

Revision : 0

Date: August 2003

\section{SAMPLE DELIVERY GROUP}

V1894 
CLOSURE RE PORT - CAU 346

Section: Appendix A

Revision : 0

Date: August 2003

\section{THIS PAGE INTENTIONALLY LEFT BLANK}




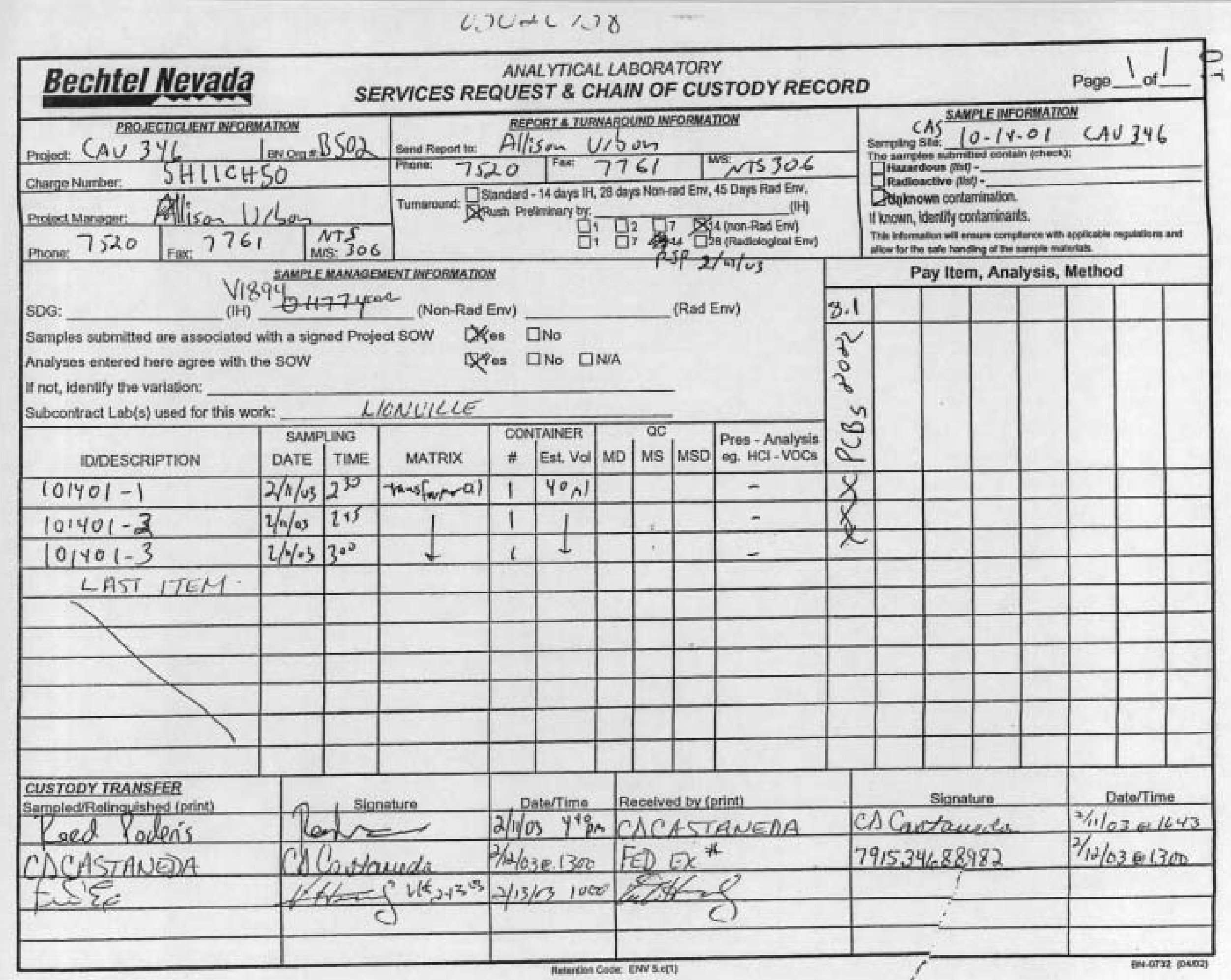


Lionville Laboratory, Inc.

PCBs by GC

RFW Barch Number: 0302L738

Client: BaCHTE, NEVADA V1894

Report Date: 02/21/03 $14: 23$ Work Order: 60052001001 Page: 1

Cust ID:

101401-1

101401-1

101401-1

101401-2

101401-3

PBLREE

Sample

Information

$\begin{array}{rc}\text { RFW\# : } & 001 \\ \text { Matrix: } & \text { OIL } \\ \text { D.P.: } & 1.00 \\ \text { Units: } & \text { OG/KG }\end{array}$

001 MS
OIL
1.00
UG/KG

001 MSD

OIL
UG/KG

002
OIL
1.00
UG/KG

003

OIL

03LE0169-MB1

UG/KG

UG/KG

$\mathrm{UG} / \mathrm{KG}$

1.00

$\mathrm{UG} / \mathrm{KG}$

SOIL

1.00

$\mathrm{UG} / \mathrm{KG}$

\begin{tabular}{|c|c|c|c|c|c|c|c|c|c|c|c|c|c|}
\hline \multirow[t]{2}{*}{ Surrogate: } & Tetrachloro-m-xylene & 55 & $\$$ & 60 & 3 & 60 & 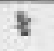 & 65 & $\xi$ & 65 & t & 100 & $\boldsymbol{t}$ \\
\hline & Decachlorobiphenyl & 115 & + & $125 *$ & 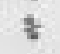 & 120 & + & 120 & 3 & 115 & 8 & $125 *$ & * $\mathbf{v}$ \\
\hline \multicolumn{14}{|c|}{ 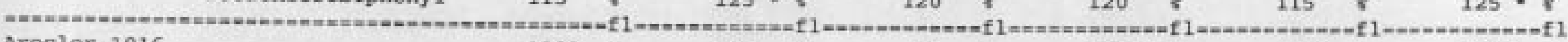 } \\
\hline Aroclor-1016 & & 1000 & $\mathrm{U}$ & 105 & 8 & 77 & 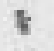 & 1000 & ¿ & 1000 & u & 1000 & 0 \\
\hline Aroclor -1221 & & 2000 & $\mathrm{u}$ & 2000 & t & 2000 & 0 & 2000 & $\mathrm{U}$ & 2000 & U & 2000 & $\mathbf{0}$ \\
\hline Aroclor -1232 & & 1000 & $\mathrm{U}$ & 1000 & $\mathrm{t}$ & 1000 & $\mathrm{U}$ & 1000 & $\mathrm{u}$ & 1000 & 0 & 1000 & $\mathbf{u}$ \\
\hline Aroclor -1242 & & 1000 & U & 1000 & $\mathrm{U}$ & 1000 & $\mathrm{U}$ & 1000 & $\mathrm{U}$ & 1000 & $\mathbf{u}$ & 1000 & t \\
\hline Aroclor-1248 & & 1000 & $\mathrm{U}$ & 1000 & $\mathrm{v}$ & 1000 & $\mathrm{v}$ & 1000 & $\mathrm{U}$ & 1000 & $\mathrm{U}$ & 1000 & U \\
\hline Aroclor -1254 & & 1000 & $\mathrm{u}$ & 1000 & $\mathrm{U}$ & 1000 & $\mathrm{v}$ & 1000 & $\mathrm{v}$ & 1000 & $\mathrm{u}$ & 1000 & $\mathrm{U}$ \\
\hline Aroclor -1260 & & 1000 & U & 105 & 3 & 107 & 8 & 1800 & & 1900 & & 1000 & $\mathrm{v}$ \\
\hline
\end{tabular}

\section{Cust ID: PBLKoE BS}

Sample

Information

$\begin{array}{rc}\text { RFFH : } & 03 \text { LE0169-MB1 } \\ \text { Matrix: } & \text { SOIL } \\ \text { D.F.: } & 1.00 \\ \text { Units: } & \text { UG/KG }\end{array}$

\begin{tabular}{|c|c|c|c|}
\hline Surrogate: & $\begin{array}{r}\text { Tetrachloro-m-xylene } \\
\text { Decachlorobiphenyl }\end{array}$ & $\begin{array}{l}105 \\
135\end{array}$ & $\begin{array}{l}z \\
z\end{array}$ \\
\hline \multicolumn{4}{|c|}{ " } \\
\hline Aroclor-1016 & & 101 & 8 \\
\hline Aroclor -1221 & & 2000 & U \\
\hline Aroclor -1232 & & 1000 & t \\
\hline Aroclor -1242 & & 1000 & $\mathrm{U}$ \\
\hline Aroclor -1248 & & 1000 & $\mathrm{U}$ \\
\hline Aroclor -1254 & & 2000 & U \\
\hline Aroclor -1260 & & 104 & 난 \\
\hline
\end{tabular}

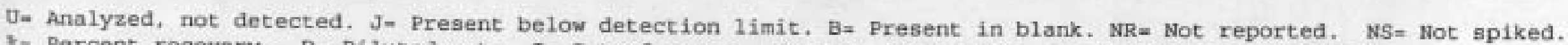
$I=$ Percent recovery. D = Diluted out. I- Interference. NAm Not Applicable. $* m$ Outgide of BPA CLP QC 


\section{APPENDIX B}

\section{SECTORED HOUSEKEEPING SITE CLOSURE VERIFICATION FORMS}


CLOSURE RE PORT - CAU 346

Section: Appendix B

Revision : 0

Date: August 2003

\section{THIS PAGE INTENTIONALLY LEFT BLANK}




\section{Sectored Housekeeping Site Closure Verification Form}

Closure Verification Date: 5/30/2003

CAU Number: 346

CAS Number: 08-22-04

CAS Description: Drums (2)

Sector Designation: NTS Sector F

Housekeeping Site General Location: Nevada Test Site, U8d

Elevation: 1,391 meters (m)

Northing: 4,114,252 m (UTM Zone: 11) Easting: 580,139 m (UTM Zone: 11)

Latitude: $37.17306 \quad$ Longitude: -116.09731

Coordinate/Elevation Data Obtained from: North American Datum, 1927.

Site Access Route: Take M ercury Highway north to Rainier Mesa Road. Turn left (northwest) on Rainier Mesa Road and proceed to 2-07 Road. Turn right (east) on 2-07 Road and proceed approximately 0.65 mile to an abandoned substation at the corner of 2-07 Road and 8-D Road. Turn left on 8-D Road and proceed 0.3 mile to a fork in the road. Take the left fork and proceed 0.25 mile to the second power line road (northwest). Follow the power line road 0.2 mile to a mud pit on the left. The site is $145 \mathrm{ft}$ south of the road, on the far side of the mud pit.

\begin{tabular}{|l|l|}
\hline \multicolumn{1}{|c|}{ Waste Item(s) Originally at Site } & \multicolumn{1}{c|}{ Apparent Waste Type* } \\
\hline Two drums & None - waste previously removed. \\
\hline
\end{tabular}

* Ordinary, Scra p Metal, Asbesto s, PCB, Salvagea ble, Hazardous, Radioactive, Mixed, Unknown, Other

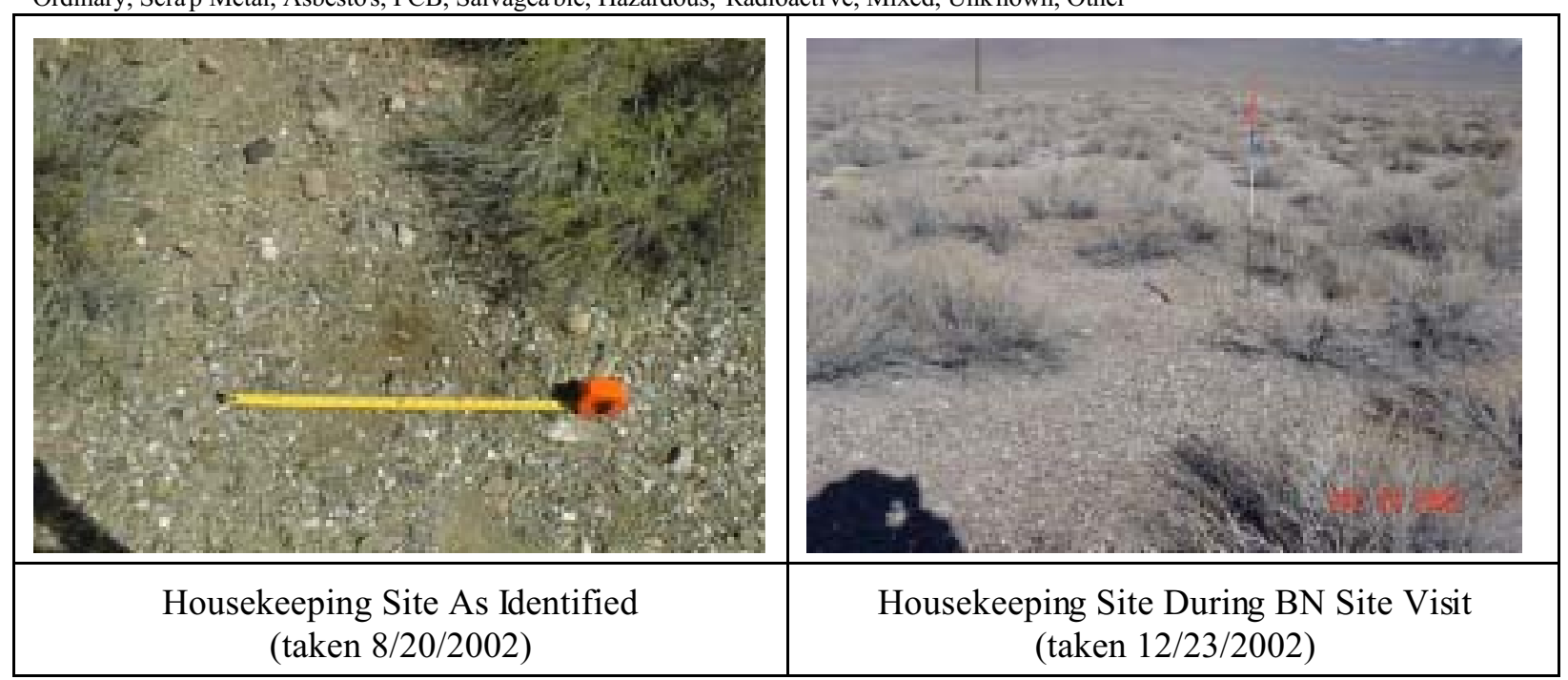

Current Site Description/Observations: The waste had been previously removed from the site.

X No Further Action Required at Housekeeping Site

Reed J. Poderis, CEM

Corrective Action Coordinator/Designee

Signature

Date 


\section{Sectored Housekeeping Site Closure Verification Form}

Closure Verification Date: 5/30/2003

CAU Number: 346

CAS Number: 08-22-11

CAS Description: Drums; Buckets

Sector Designation: NTS Sector F

Housekeeping Site General Location: Nevada Test Site, U8d

Elevation: 1,396 meters (m)

Northing: 4,114,183 m (UTM Zone: 11) Easting: 579,846 m (UTM Zone: 11)

Latitude: $37.17248 \quad$ Longitude: -116.10065

Coordinate/Elevation Data Obtained from: North American Datum, 1927.

Site Access Route: Take Mercury Highway north to Rainier Mesa Road. Turn left (northwest) on Rainier Mesa Road and proceed to 2-0 7 Road. Turn right (e ast) on 2-07 Road and proceed $0.2 \mathrm{~m}$ ile to RSM 2L-13. Turn left (north) on the dirt road immediately past RSM 2L-13 and proceed 0.75 mile to the south side of the U-8d Crater. The drums are in the pits on the south side of $U-8 \mathrm{~d}$.

\begin{tabular}{|l|l|}
\hline \multicolumn{1}{|c|}{ Waste Item(s) Originally at Site } & \multicolumn{1}{c|}{ Apparent Waste Type* } \\
\hline Drums, buckets & Ordinary \\
\hline
\end{tabular}

* Ordinary, Scrap Metal, Asbesto s, PCB, Salvageable, Hazardous, Radioactive, Mixed, Unk nown, Other

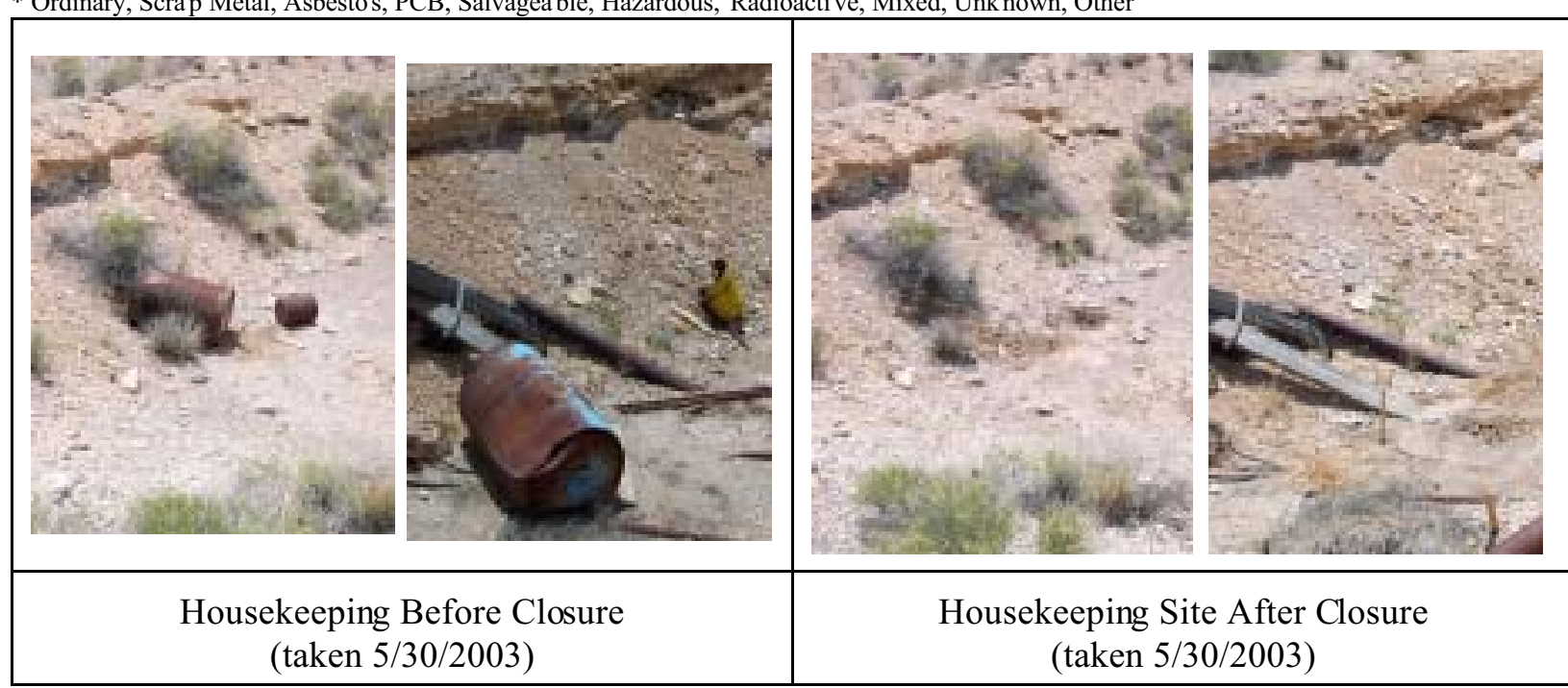

Current Site Description/Observations: The empty drums and buckets were disposed of at the Area 9

U10c Landfill.

X No Further Action Required at Housekeeping Site

Reed J. Poderis, CEM

Corrective Action Coordinator/Designee

Signature

Date

B-2 


\section{Sectored Housekeeping Site Closure Verification Form}

Closure Verification Date: 5/30/2003

CAU Number: 346

CAS Number: 08-24-02

CAS Description: Battery

Sector Designation: NTS Sector F

Housekeeping Site General Location: Nevada Test Site, U8c

Elevation: 1,350 meters $(\mathrm{m})$

Northing: 4,114,069 m (UTM Zone: 11) Easting: 581,863 m (UTM Zone: 11)

Latitude: $37.17127 \quad$ Longitude: -116.07792

Coordinate/Elevation Data Obtained from: North American Datum, 1927.

Site Access Route: Take Mercury Highway north to Rainier Mesa Road. Turn left (northwest) on Rainier Mesa Road and proceed to 2-07 Road. Turn right (east) on 2-07 Road and proceed approximately $1.0 \mathrm{mile}$ to a dirt road on the left (north). Turn left on the dirt road and continue 0.3 mile to a power line road. The U-8c Crater area is on the northwest corner of this intersection. T urn right (east) onto the pow er line road and travel 0.5 mile. The site is located in a slight wash area on the right (south) side of the powering road.

\begin{tabular}{|l|l|}
\hline \multicolumn{1}{|c|}{ Waste Item(s) Originally at Site } & \multicolumn{1}{c|}{ Apparent Waste Type* $^{*}$} \\
\hline Battery & None - waste previously removed. \\
\hline
\end{tabular}

* Ordinary, Scra p Metal, Asbesto s, PCB, Salvagea ble, Hazardous, Radioactive, Mixed, Unknown, Other

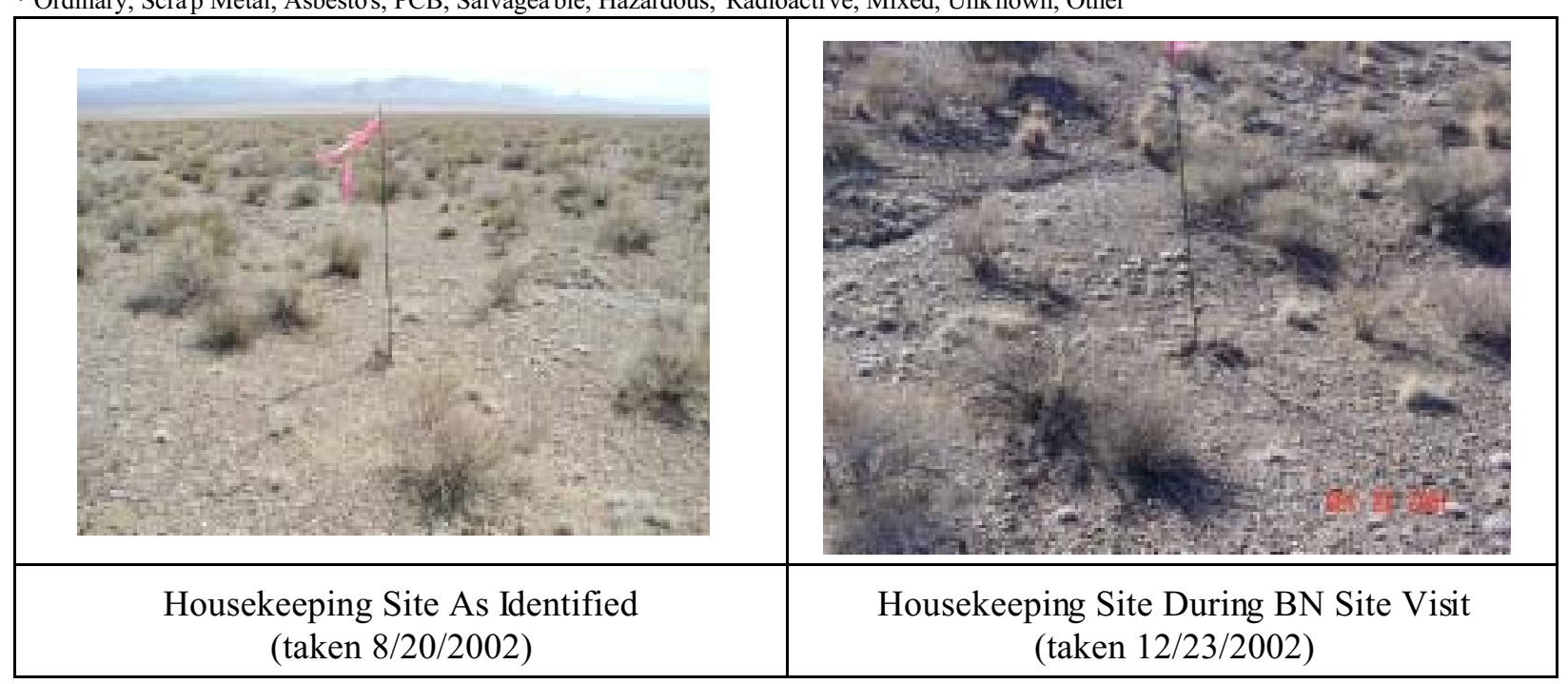

Current Site Description/Observations: The waste had been previously removed from the site.

X No Further Action Required at Housekeeping Site

Reed J. Poderis, CEM

Corrective Action Coordinator/Designee

Signature

Date 


\section{Sectored Housekeeping Site Closure Verification Form}

Closure Verification Date: 7/16/2003

CAU Number: 346

CAS Number: 10-14-01

CAS Description: Transformer

Sector Designation: NTS Sector F

Housekeeping Site General Location: Nevada Test Site, U10am \#5

Elevation: 1,341 meters (m)

Northing: 4,116,304 m (UTM Zone: 11) Easting: 585,685 m (UTM Zone: 11)

Latitude: $37.19109 \quad$ Longitude: -116.03465

Coordinate/Elevation Data Obtained from: North American Datum, 1927.

Site Access Route: Take M ercury Highway north to Rainier Mesa Road. Turn left (northwest) onto Rainier Mesa Road and proceed to 2-07 Road. Turn right (east) and proceed to the Circle Road intersection. Turn left (north) on Circle Road and proceed to 10-02 Road. Turn left (north) onto 10-02 Road and continue to the power line road. Turn right (east) at the power line road and proceed approximately 0.65 mile. The site is located $50 \mathrm{ft}$ north of the power line road and $150 \mathrm{ft}$ southwest from the U-10cb Crater.

\begin{tabular}{|l|l|}
\hline \multicolumn{1}{|c|}{ Waste Item(s) Originally at Site } & \multicolumn{1}{|c|}{ Apparent Waste Type* } \\
\hline Oil-Filled Circuit Breaker & Salvageable \\
\hline
\end{tabular}

* Ordinary, Scra p Metal, Asbesto s, PCB, Salvagea ble, Hazardous, Radioactive, Mixed, Unknown, Other

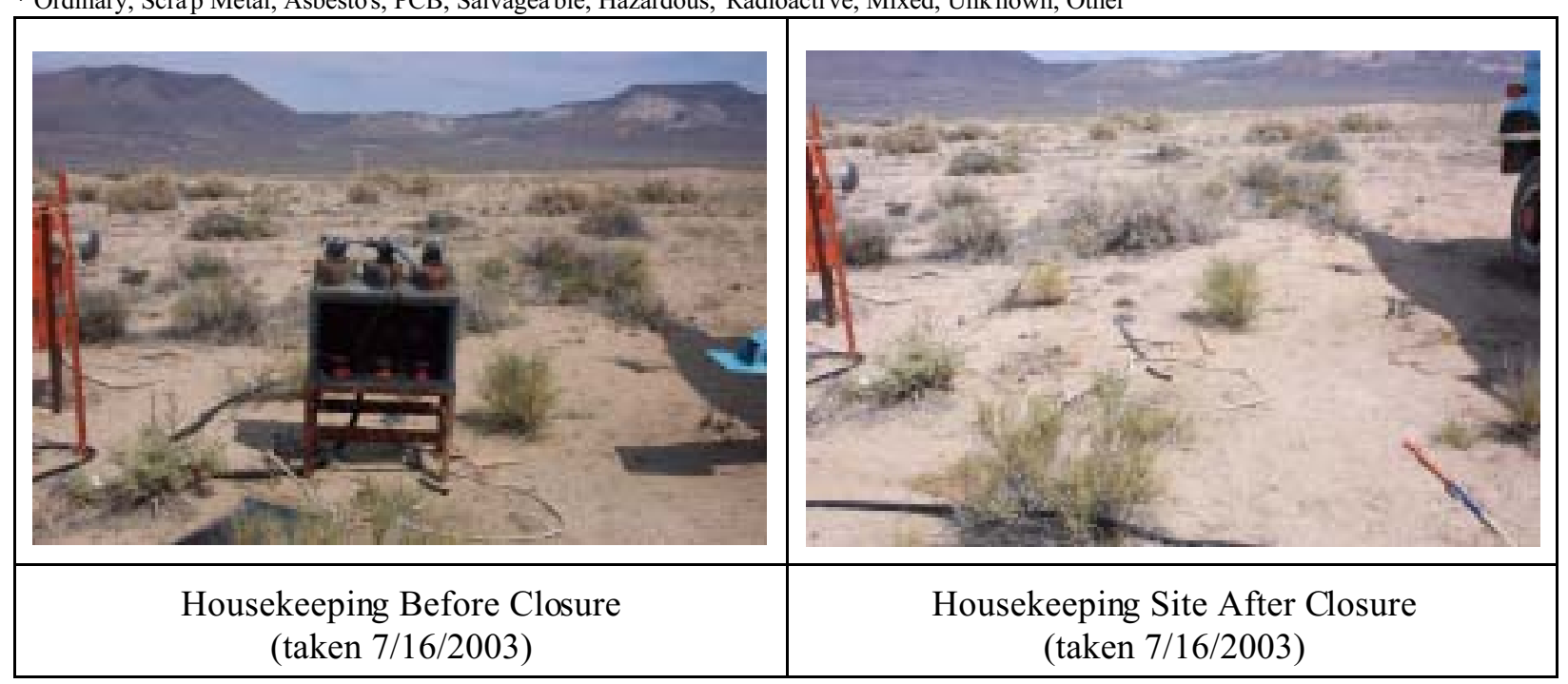

Current Site Description/Observations: The circuit breaker was salvaged by Site Utilities.

X No Further Action Required at Housekeeping Site

Reed J. Poderis, CEM

Corrective Action Coordinator/Designee

Signature

Date 


\section{Sectored Housekeeping Site Closure Verification Form}

Closure Verification Date: 5/30/2003

CAU Number: 346

CAS Number: 10-22-06

CAS Description: Drum (Gas Block)

Sector Designation: NTS Sector F

Housekeeping Site General Location: Nevada Test Site, U10s

Elevation: 1,304 meters (m)

Northing: 4,113,796 m (UTM Zone: 11) Easting: 584,485 m (UTM Zone: 11)

Latitude: $37.16855 \quad$ Longitude: -116.04835

Coordinate/Elevation Data Obtained from: North American Datum, 1927.

Site Access Route: Take M ercury Highway north to Rainier Mesa Road. Turn left (northwest) onto Rainier Mesa Road and proceed to 2-07 Road. Turn right (east) and proceed to the Circle Road intersection. Turn left (north) on Circle Road and proceed 0.7 mile to 10-01 Road. Turn right (east) on 10-01 Road and proceed 0.85 mile. Turn right (south) and proceed cross country to the U-10s Crater. The site is $18 \mathrm{ft}$ east of the crater fence line.

\begin{tabular}{|l|l|}
\hline \multicolumn{1}{|c|}{ Waste Item(s) Originally at Site } & \multicolumn{1}{c|}{ Apparent Waste Type* } \\
\hline 55-Gallon Drum Gas Block & Ordinary \\
\hline
\end{tabular}

* Ordinary, Scra p Metal, Asbesto s, PCB, Salvagea ble, Hazardous, Radioacti ve, Mixed, Unknown, Other

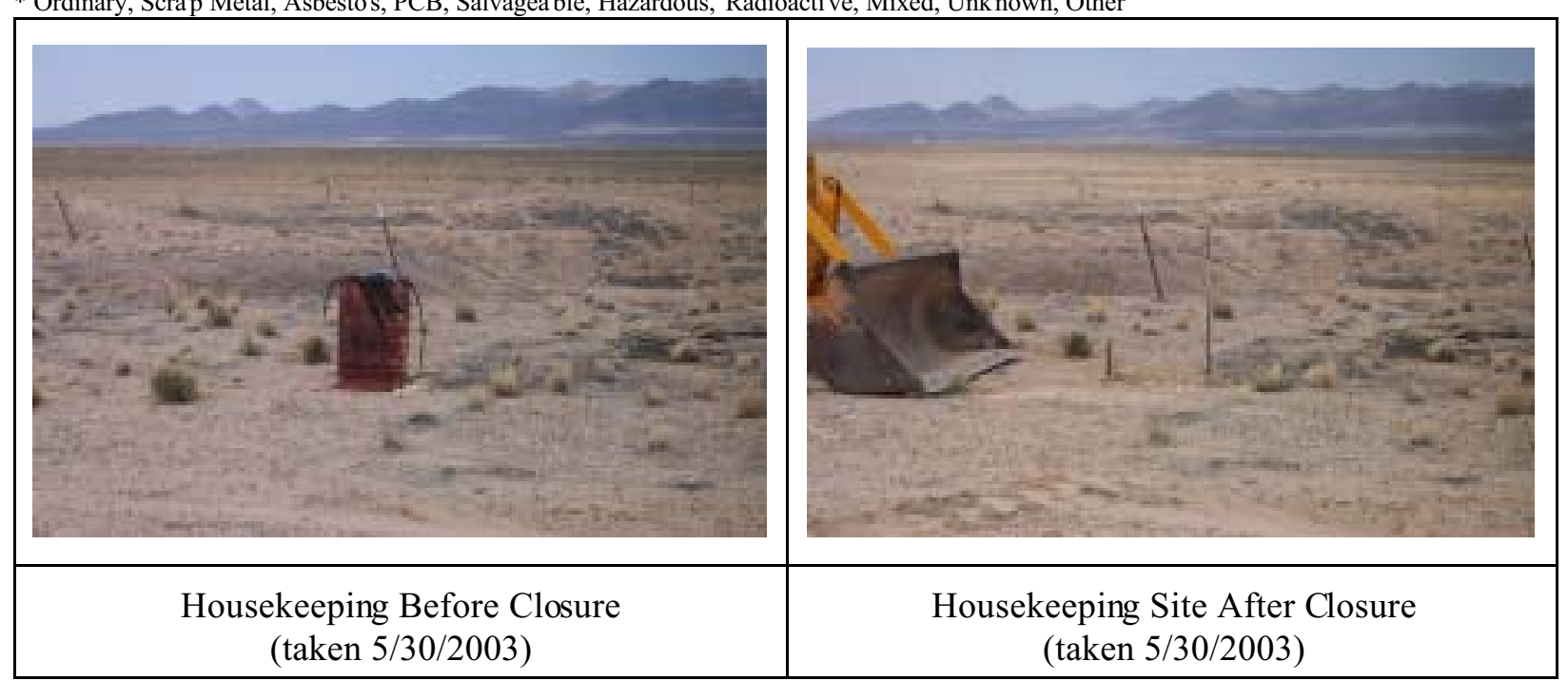

Current Site Description/Observations: The drum gas block was disposed of in the Area 9 U10c Landfill.

X No Further Action Required at Housekeeping Site

Reed J. Poderis, CEM

Corrective Action Coordinator/Designee

Signature

Date 


\section{Sectored Housekeeping Site Closure Verification Form}

Closure Verification Date: 5/30/2003

CAU Number: 346

CAS Number: 10-22-10

CAS Description: Drum (Gas Block)

Sector Designation: NTS Sector F

Housekeeping Site General Location: Nevada Test Site, U10ajf

Elevation: 1,307 meters (m)

Northing: 4,113,600 m (UTM Zone: 11) Easting: 584,949 m (UTM Zone: 11)

Latitude: $37.16675 \quad$ Longitude: -116.04319

Coordinate/Elevation Data Obtained from: North American Datum, 1927.

Site Access Route: Take M ercury Highway north to Rainier Mesa Road. Turn left (northwest) onto Rainier Mesa Road and proceed to 2-07 Road. Turn right (east) and proceed to the Circle Road intersection. Turn left (north) on Circle Road and proceed 0.7 mile to 10-01 Road. Turn right (east) onto 10-01 Road and proceed

1.2 miles to the Teapot area. Turn right (south) at the bridge structure and proceed a pproxim ately $800 \mathrm{ft}$ to the site.

The site is $65 \mathrm{ft}$ southwest of the U10-AUA Borehole.

\begin{tabular}{|l|l|}
\hline \multicolumn{1}{|c|}{ Waste Item(s) Originally at Site } & \multicolumn{1}{|c|}{ Apparent Waste Type* } \\
\hline 55-Gallon Drum Gas Block & Ordinary \\
\hline
\end{tabular}

* Ordinary, Scrap Metal, Asbesto s, PCB, Salvagea ble, Hazardous, Radioactive, Mixed, Unknown, Other

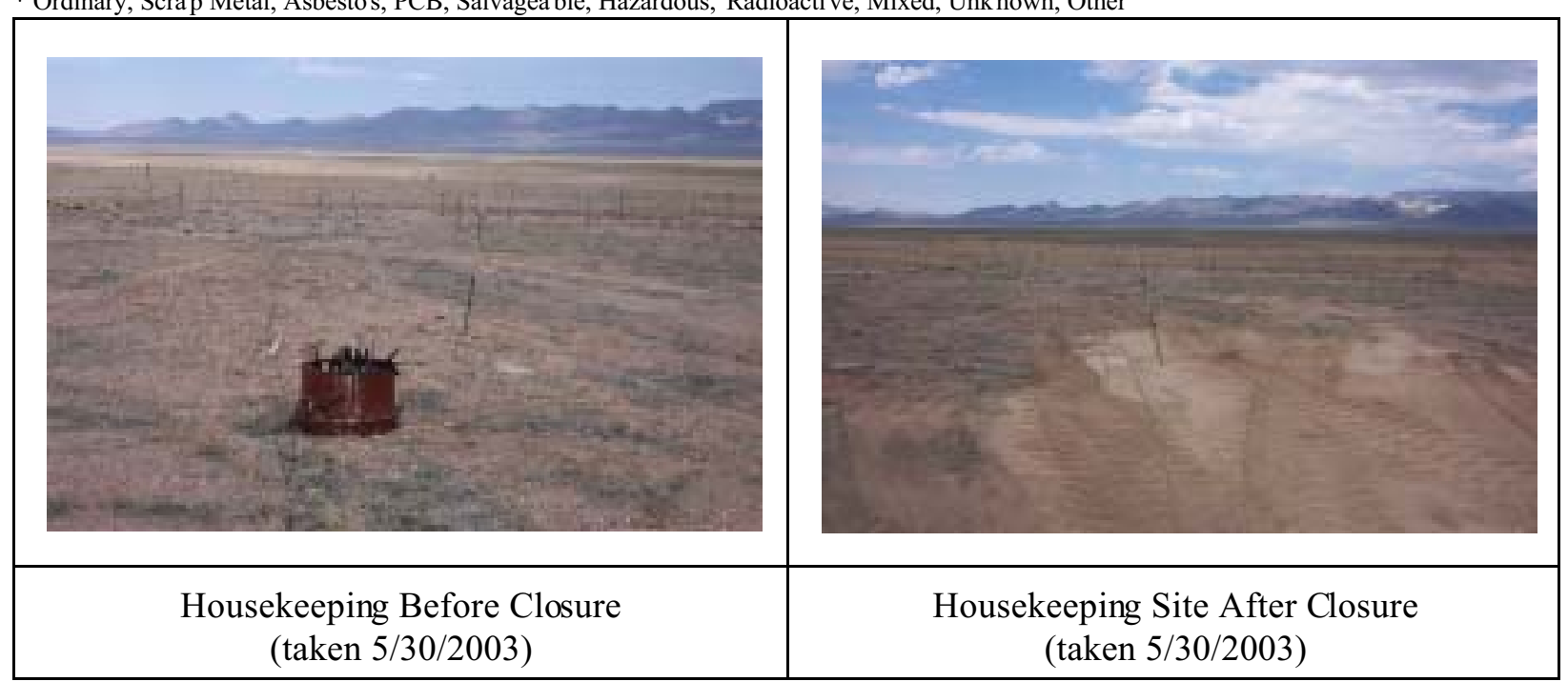

Current Site Description/Observations: The drum gas block was disposed of in the Area 9 U10c Landfill.

$\underline{X}$ No Further Action Required at Housekeeping Site

Reed J. Poderis, CEM

Corrective Action Coordinator/Designee

Signature

Date 


\section{Sectored Housekeeping Site Closure Verification Form}

Closure Verification Date: 5/30/2003

CAU Number: 346

CAS Number: 10-22-12

CAS Description: Drum (Gas Block)

Sector Designation: NTS Sector F

Housekeeping Site General Location: Nevada Test Site, U10ajf

Elevation: 1,310 meters (m)

Northing: 4,113,514 m (UTM Zone: 11) Easting: 585,163 m (UTM Zone: 11)

Latitude: $37.16599 \quad$ Longitude: -116.04079

Coordinate/Elevation Data Obtained from: North American Datum, 1927.

Site Access Route: Take M ercury Highway north to Rainier Mesa Road. Turn left (northwest) onto Rainier Mesa Road and proceed to 2-07 Road. Turn right (east) and proceed to the Circle Road intersection. Turn left (north) on Circle Road and proceed 0.7 mile to 10-01 Road. Turn right (east) on 10-01 Road and proceed 1.2 miles to the Teapot area. Turn right (southeast) and travel cross country to the U-10aj F Potential Crater. This site is $380 \mathrm{ft}$ northeast of U-10aj F.

\begin{tabular}{|l|l|}
\hline \multicolumn{1}{|c|}{ Waste Item(s) Originally at Site } & \multicolumn{1}{c|}{ Apparent Waste Type* } \\
\hline 55-Gallon Drum Gas Block & Ordinary \\
\hline
\end{tabular}

* Ordinary, Scra p Metal, Asbesto s, PCB, Salvagea ble, Hazardous, Radioactive, Mixed, Unknown, Other

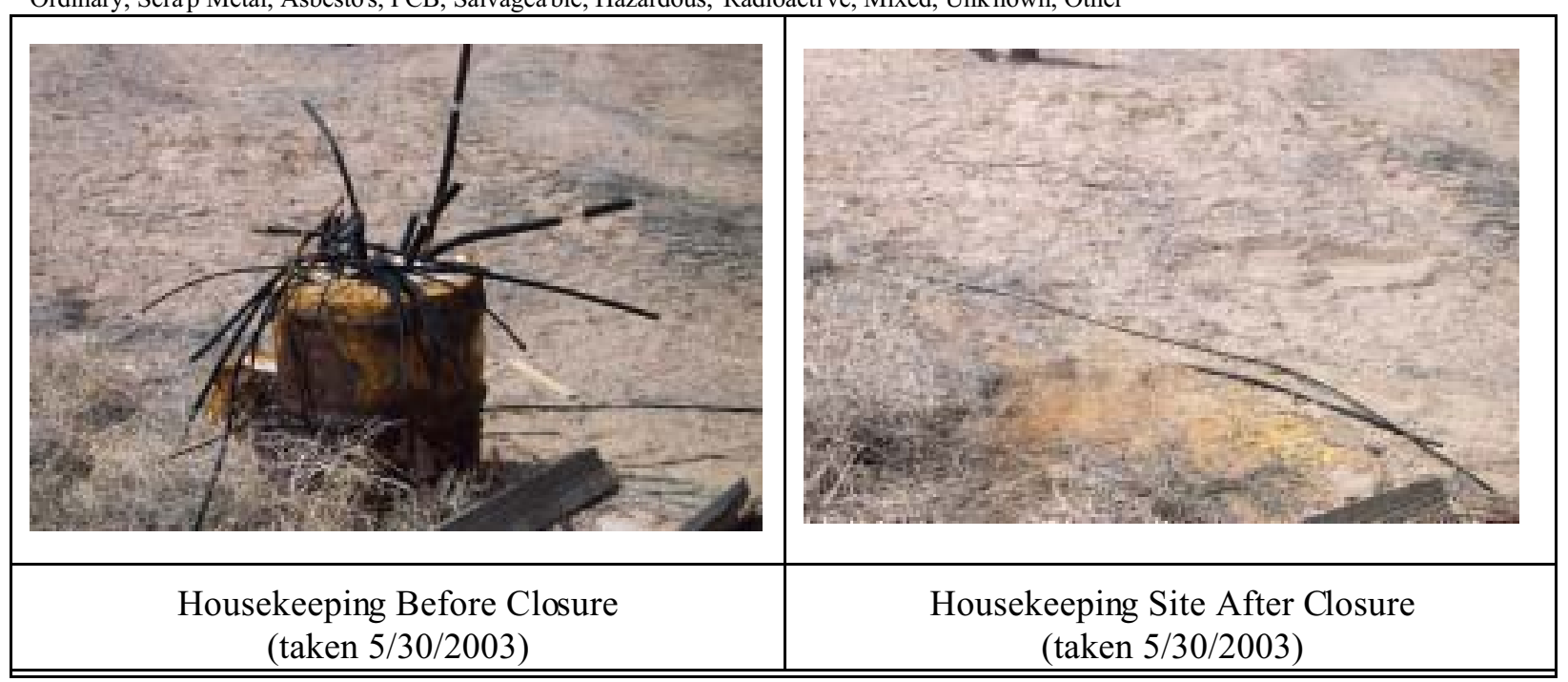

Current Site Description/Observations: The drum gas block was disposed of in the Area 9 U10c Landfill.

X No Further Action Required at Housekeeping Site

Reed J. Poderis, CEM

Corrective Action Coordinator/Designee

Signature

Date 


\section{Sectored Housekeeping Site Closure Verification Form}

Closure Verification Date: 5/30/2003

CAU Number: 346

CAS Number: 10-22-13

CAS Description: Drum (Gas Block)

Sector Designation: NTS Sector F

Housekeeping Site General Location: Nevada Test Site, U10ag

Elevation: 1,310 meters (m)

Northing: 4,113,503 m (UTM Zone: 11) Easting: 585,163 m (UTM Zone: 11)

Latitude: $37.16588 \quad$ Longitude: -116.04077

Coordinate/Elevation Data Obtained from: North American Datum, 1927.

Site Access Route: Take M ercury Highway north to Rainier Mesa Road. Turn left (northwest) onto Rainier Mesa Road and proceed to 2-07 Road. Turn right (east) and proceed to the Circle Road intersection. Turn left (north) on Circle Road and proceed 0.7 mile to 10-01 Road. Turn right (east) on 10-01 Road and proceed 1.2 miles to the Teapot area. Turn right (southeast) and travel cross country to the U-10ag Potential Crater. The site is $350 \mathrm{ft}$ northeast of U-10ag.

\begin{tabular}{|l|l|}
\hline \multicolumn{1}{|c|}{ Waste Item(s) Originally at Site } & \multicolumn{1}{c|}{ Apparent Waste Type* $^{*}$} \\
\hline 55-Gallon Drum Gas Block & Ordinary \\
\hline
\end{tabular}

* Ordinary, Scra p Metal, Asbesto s, PCB, Salvagea ble, Hazardous, Radioactive, Mixed, Unknown, Other

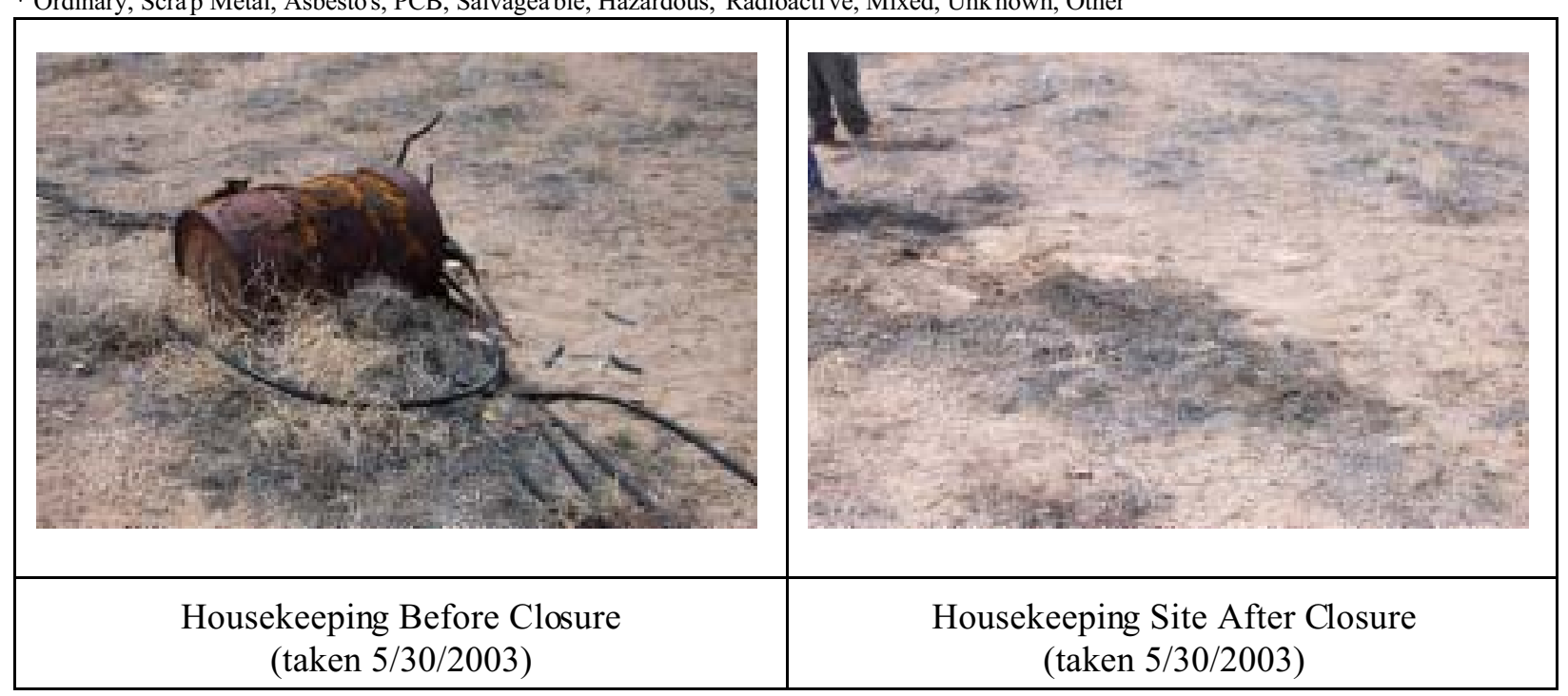

Current Site Description/Observations: The drum gas block was disposed of in the Area 9 U10c Landfill.

X No Further Action Required at Housekeeping Site

Reed J. Poderis, CEM

Corrective Action Coordinator/Designee

Signature

Date 


\section{Sectored Housekeeping Site Closure Verification Form}

Closure Verification Date: 5/30/2003

CAU Number: 346

CAS Number: 10-22-16

CAS Description: Drum (Gas Block)

Sector Designation: NTS Sector F

Housekeeping Site General Location: Nevada Test Site, U10m

Elevation: 1,307 meters $(\mathrm{m})$

Northing: 4,112,549 m (UTM Zone: 11) Easting: 585,243 m (UTM Zone: 11)

Latitude: $37.15733 \quad$ Longitude: -116.04002

Coordinate/Elevation Data Obtained from: North American Datum, 1927.

Site Access Route: Take M ercury Highway north to Rainier Mesa Road. Turn left (northwest) onto Rainier Mesa Road and proceed to 2-05 Road. Turn right (east) onto 2-05 Road and proceed to Circle Road. At the Circle Road intersection, continue straight on the dirt road 0.85 mile to a " $\mathrm{V}$ " in the road. Turn left onto an unmaintained dirt road and proceed 0.5 mile. Turn left (north) and drive cross country 0.2 mile to the U- $10 \mathrm{~m}$ Potential Crater.

\begin{tabular}{|l|l|}
\hline \multicolumn{1}{|c|}{ Waste Item(s) Originally at Site } & \multicolumn{1}{c|}{ Apparent Waste Type* } \\
\hline 55-Gallon Drum Gas Block & Ordinary \\
\hline
\end{tabular}

* Ordinary, Scrap Metal, Asbesto s, PCB, Salvagea ble, Hazardous, Radioacti ve, Mixed, Unk nown, Other

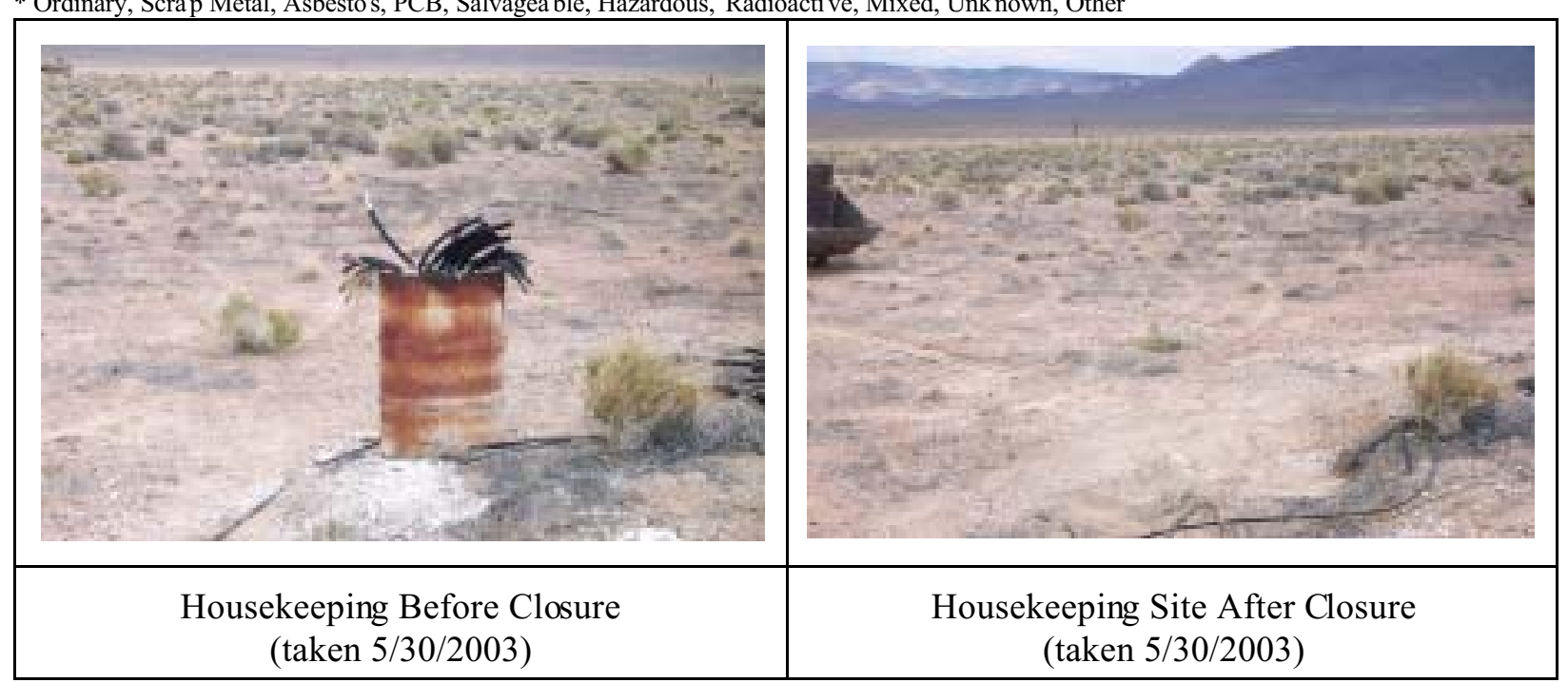

Current Site Description/Observations: The drum gas block was disposed of in the Area 9 U10c Landfill.

X No Further Action Required at Housekeeping Site

Reed J. Poderis, CEM

Corrective Action Coordinator/Designee

Signature

Date 


\section{Sectored Housekeeping Site Closure Verification Form}

Closure Verification Date: 5/30/2003

CAU Number: 346

CAS Number: 10-22-22

CAS Description: Drum

Sector Designation: NTS Sector F

Housekeeping Site General Location: Nevada Test Site, U10am \#3

Elevation: 1,348 meters $(\mathrm{m})$

Northing: 4,113,759 m (UTM Zone: 11) Easting: 581,901 m (UTM Zone: 11)

Latitude: $37.1685 \quad$ Longitude: -116.07751

Coordinate/Elevation Data Obtained from: North American Datum, 1927.

Site Access Route: Take M ercury Highway north to Rainier Mesa Road. Turn left (northwest) on Rainier Mesa Road and proceed to 2-07 Road. Turn right (e ast) and proceed 1.35 miles to an unmaintained road on the left, $600 \mathrm{ft}$ before an old junction box. Turn left (north) on the acc ess road and follow the ro ad as it curves east 0.25 mile around the U-10am \#3 Crater to the site.

\begin{tabular}{|l|l|}
\hline \multicolumn{1}{|c|}{ Waste Item(s) Originally at Site } & \multicolumn{1}{c|}{ Apparent Waste Type* $^{*}$} \\
\hline 40-Gallon Drum & Ordinary \\
\hline
\end{tabular}

* Ordinary, Scra p Metal, Asbesto s, PCB, Salvagea ble, Hazardous, Radioactive, Mixed, Unk nown, Other

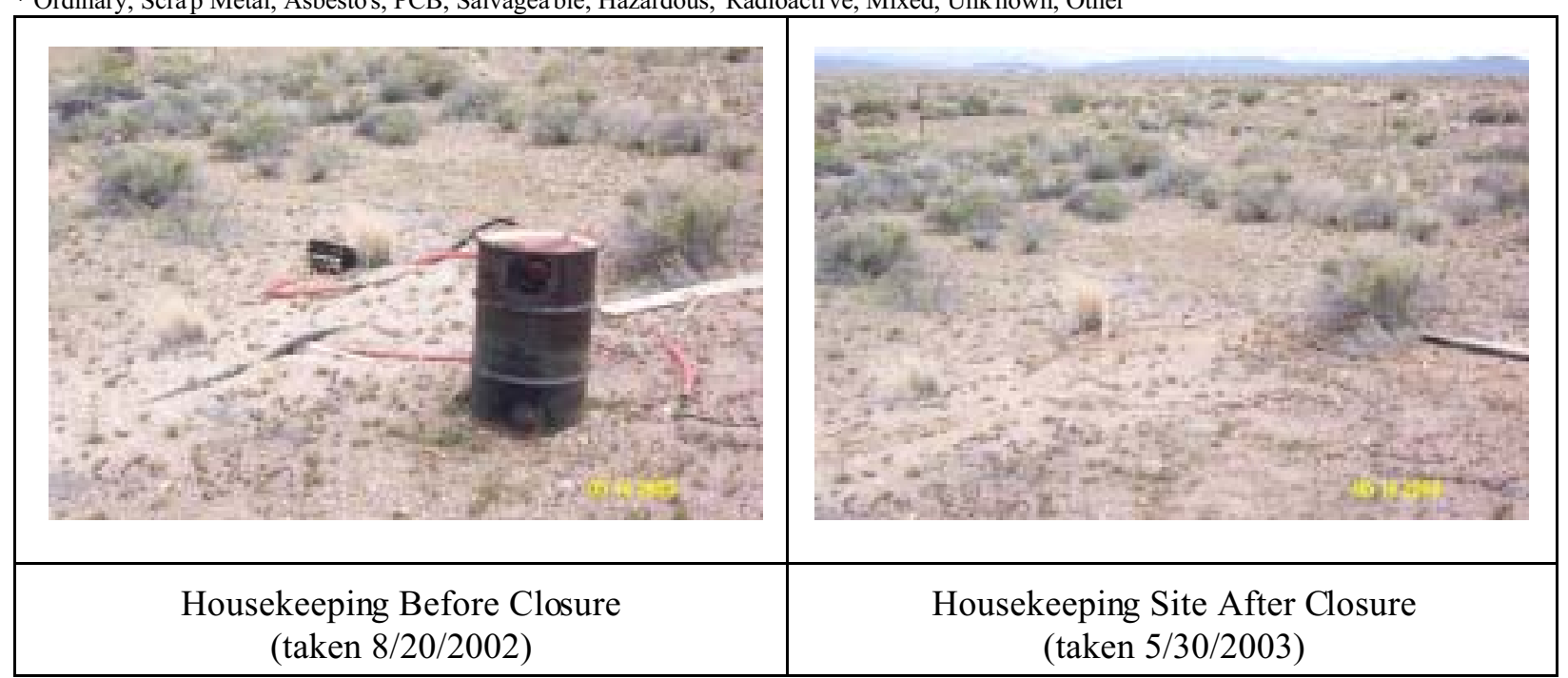

Current Site Description/Observations: The drum was disposed of in the Area 9 U10c Landfill.

X No Further Action Required at Housekeeping Site

Reed J. Poderis, CEM

Corrective Action Coordinator/Designee

Signature

Date 


\section{Sectored Housekeeping Site Closure Verification Form}

Closure Verification Date: 5/30/2003

CAU Number: 346

CAS Number: 10-22-25

CAS Description: Drum

Sector Designation: NTS Sector F

Housekeeping Site General Location: Nevada Test Site, U10am \#1

Elevation: 1,353 meters $(\mathrm{m})$

Northing: 4,113,745 m (UTM Zone: 11) Easting: 581,653 m (UTM Zone: 11)

Latitude: $37.16841 \quad$ Longitude: -116.08031

Coordinate/Elevation Data Obtained from: North American Datum, 1927.

Site Access Route: Take M ercury Highway north to Rainier Mesa Road. Turn left (northwest) onto Rainier Mesa Road and proceed to 2-07 Road. Turn right (east) onto 2-07 Road and continue approximately 1.4 miles to a dirt road on the left (north). T urn left on the dirt road and proceed approx imately 0.2 mile to U-10 am \#1. The site is located in a fenced area north of U-10am \#1.

\begin{tabular}{|l|l|}
\hline \multicolumn{1}{|c|}{ Waste Item(s) Originally at Site } & \multicolumn{1}{c|}{ Apparent Waste Type* } \\
\hline 40-Gallon Drum & Ordinary \\
\hline
\end{tabular}

* Ordinary, Scra p Metal, Asbesto s, PCB, Salvagea ble, Hazardous, Radioactive, Mixed, Unknown, Other

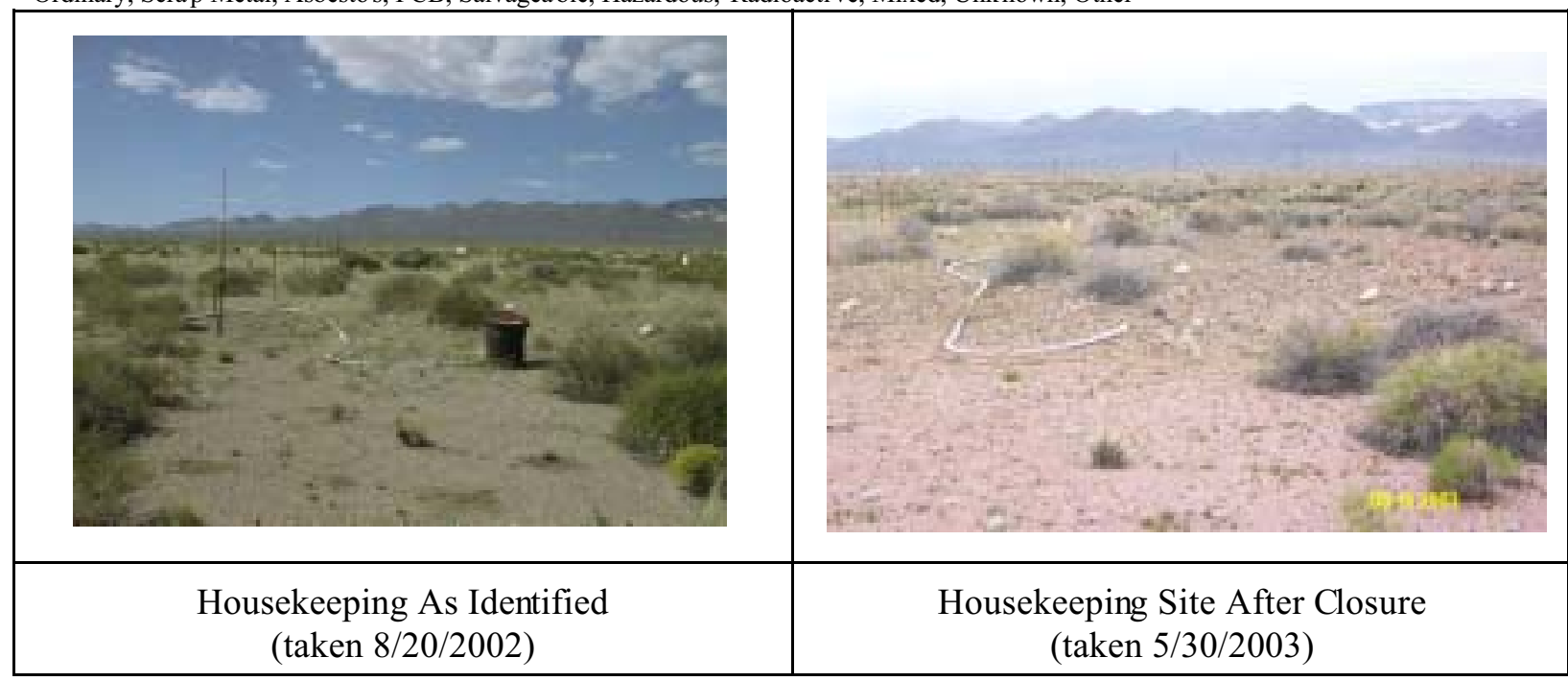

Current Site Description/Observations: The drum was disposed of in the Area 9 U10c Landfill.

X No Further Action Required at Housekeeping Site

Reed J. Poderis, CEM

Corrective Action Coordinator/Designee

Signature

Date 


\section{Sectored Housekeeping Site Closure Verification Form}

Closure Verification Date: 5/30/2003

CAU Number: 346

CAS Number: 10-22-36

CAS Description: Paint Can

Sector Designation: NTS Sector F

Housekeeping Site General Location: Nevada Test Site, U10am \#1

Elevation: 1,350 meters (m)

Northing: 4,113,580 m (UTM Zone: 11) Easting: 581,797 m (UTM Zone: 11)

Latitude: $37.16692 \quad$ Longitude: -116.07872

Coordinate/Elevation Data Obtained from: North American Datum, 1927.

Site Access Route: Take M ercury Highway north to Rainier Mesa Road. Turn left (northwest) on Rainier Mesa Road and proceed to 2-03 Road. Turn right (northeast) onto 2-03 and continue as it merges into Circle Road. Take Circle Road north to 2-07 Road. Turn left (west) onto 2-07 Road and continue 1.0 mile to the electric junction boxes on the right (north). The site is $500 \mathrm{ft}$ north $\left(346^{\circ}\right)$ of the road.

\begin{tabular}{|l|l|}
\hline \multicolumn{1}{|c|}{ Waste Item(s) Originally at Site } & \multicolumn{1}{c|}{ Apparent Waste Type* } \\
\hline Empty one-gallon Paint Can & Ordinary \\
\hline
\end{tabular}

* Ordinary, Scrap Metal, Asbesto s, PCB, Salvagea ble, Hazardous, Radioactive, Mixed, Unk nown, Other

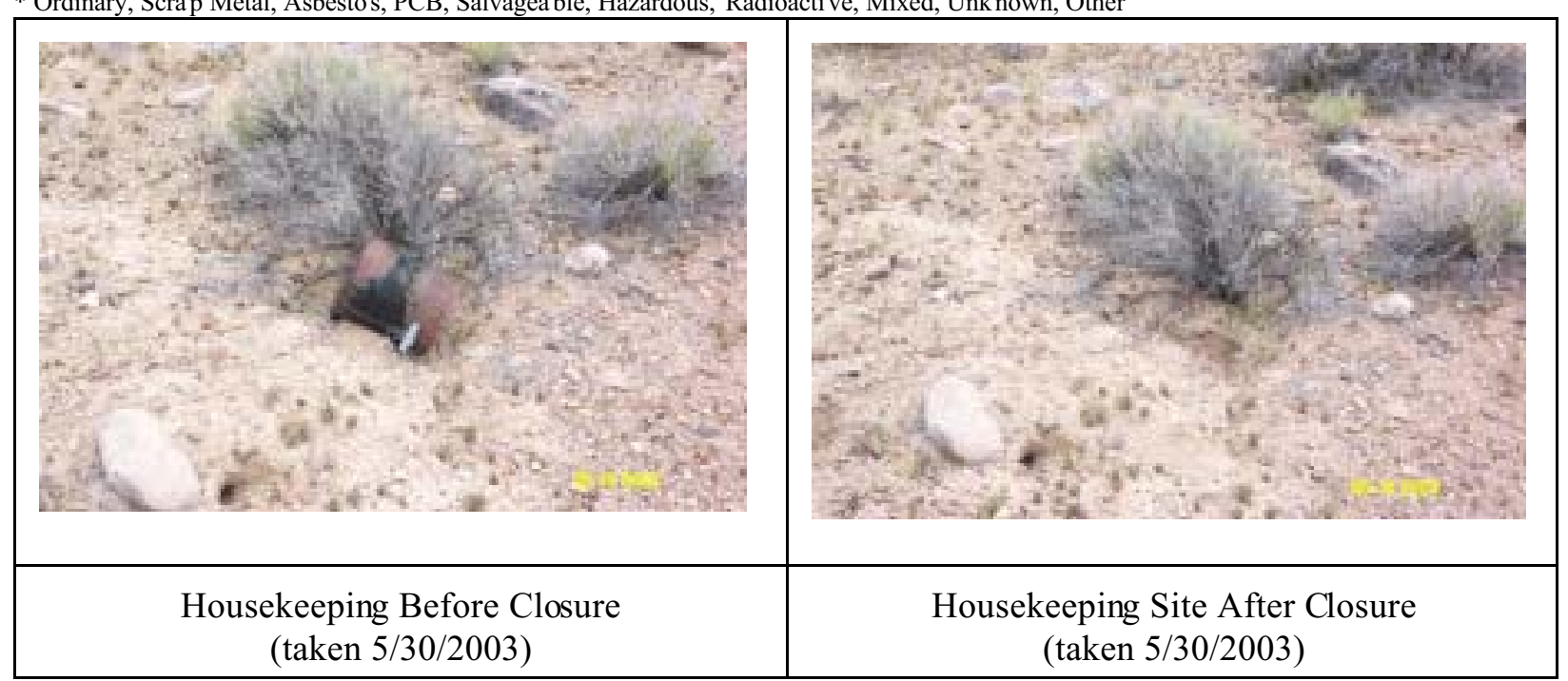

Current Site Description/Observations: The can was disposed of in the Area 9 U10c Landfill.

X No Further Action Required at Housekeeping Site

Reed J. Poderis, CEM

Corrective Action Coordinator/Designee

Signature

Date 


\section{Sectored Housekeeping Site Closure Verification Form}

Closure Verification Date: 5/30/2003

CAU Number: 346

CAS Number: 10-22-37

CAS Description: Gas Block

Sector Designation: NTS Sector F

Housekeeping Site General Location: Nevada Test Site, U10ajf

Elevation: 1,307 meters (m)

Northing: 4,113,469 m (UTM Zone: 11) Easting: 585,083 m (UTM Zone: 11)

Latitude: $37.16558 \quad$ Longitude: -116.04169

Coordinate/Elevation Data Obtained from: North American Datum, 1927.

Site Access Route: Take M ercury Highway north to Rainier Mesa Road. Turn left (northwest) onto Rainier Mesa Road and proceed to 2-07 Road. Turn right (east) and proceed to the Circle Road intersection. Turn left (north) on Circle Road and proceed 0.7 mile to 10-01 Road. Turn right (east) onto 10-01 Road and proceed 1.2 miles to the Teapot area. Turn right southeast and travel cross country to the U-10aj F Potential Crater.

\begin{tabular}{|l|l|}
\hline \multicolumn{1}{|c|}{ Waste Item(s) Originally at Site } & \multicolumn{1}{c|}{ Apparent Waste Type* } \\
\hline 55-Gallon Drum Gas Block & Ordinary \\
\hline
\end{tabular}

* Ordinary, Scrap Metal, Asbesto s, PCB, Salvagea ble, Hazardous, Radioacti ve, Mixed, Unk nown, Other

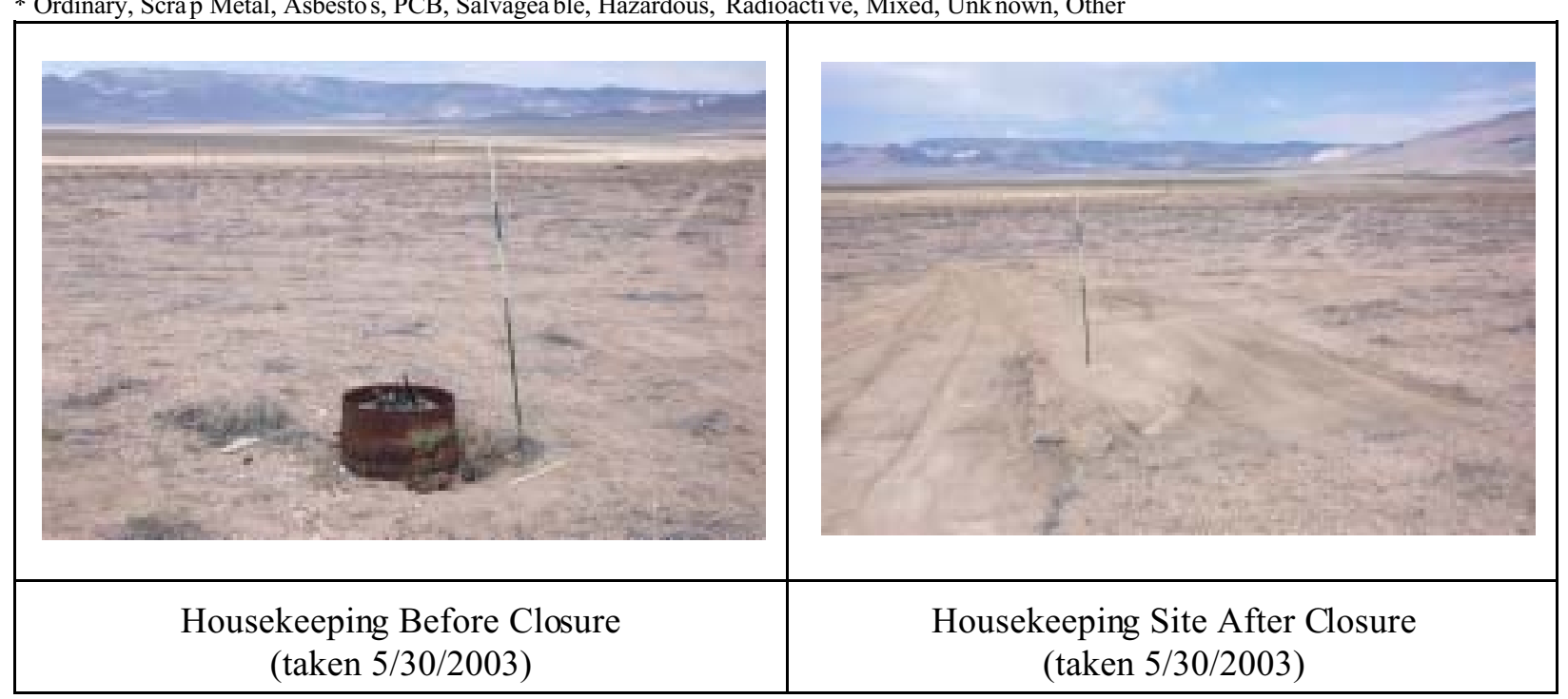

Current Site Description/Observations: The drum gas block was disposed of in the Area 9 U10c Landfill.

X No Further Action Required at Housekeeping Site

Reed J. Poderis, CEM

Corrective Action Coordinator/Designee

Signature

Date 


\section{Sectored Housekeeping Site Closure Verification Form}

Closure Verification Date: 5/30/2003

CAU Number: 346

CAS Number: 10-24-11

CAS Description: Battery

Sector Designation: NTS Sector F

Housekeeping Site General Location: Nevada Test Site, U10am \#3

Elevation: 1,348 meters (m)

Northing: 4,113,759 m (UTM Zone: 11) Easting: 581,901 m (UTM Zone: 11)

Latitude: $37.1685 \quad$ Longitude: -116.07751

Coordinate/Elevation Data Obtained from: North American Datum, 1927.

Site Access Route: Take Mercury Highway north to Rainier Mesa Road. Turn left (northwest) on Rainier Mesa Road and proceed to 2-07 Road. Turn right (e ast) and proceed 1.35 miles to an unmaintained road on the left, $600 \mathrm{ft}$. before an old junction box. Turn left (north) on the access road and follow the ro ad as it curves east 0.25 mile around the U-10am \#3 Crater to the site.

\begin{tabular}{|l|l|}
\hline \multicolumn{1}{|c|}{ Waste Item(s) Originally at Site } & \multicolumn{1}{c|}{ Apparent Waste Type* $^{*}$} \\
\hline One Intact Automotive Battery & Salvageable \\
\hline
\end{tabular}

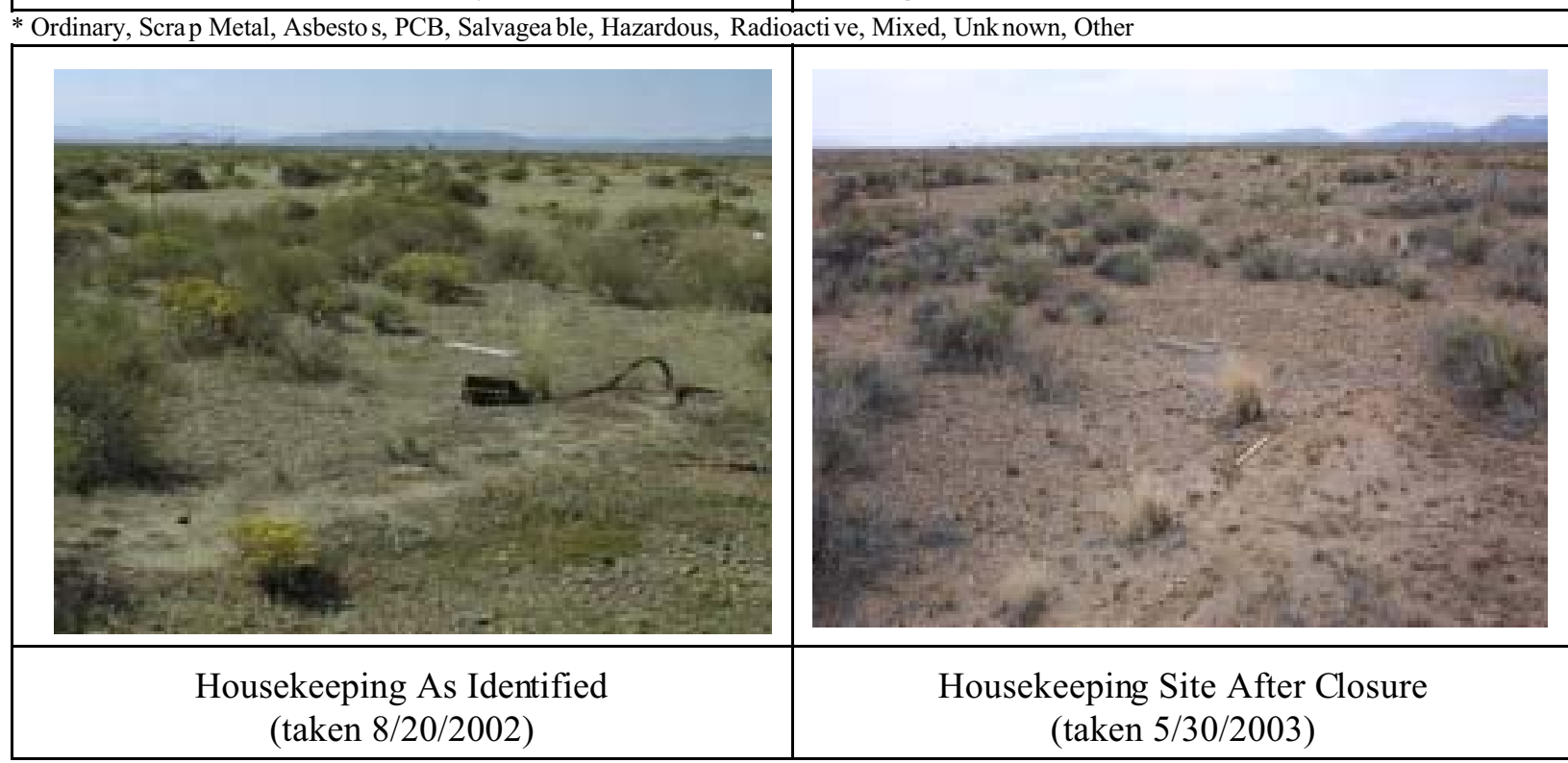

Current Site Description/Observations: The battery was recycled by BN Fleet Operations.

\section{$\underline{X}$ No Further Action Required at Housekeeping Site}

Reed J. Poderis, CEM 


\section{APPENDIX C}

\section{U.S. DEPARTMENT OF ENERGY NATIONAL NUCLEAR SECURITY ADMINISTRATION NEVADA OPERATIONS OFFICE NEPA ENVIRONMENTAL EVALUATION CHECKLIST}


CLOSURE RE PORT - CAU 346 Section: Appendix C

Revision : 0

Date: August 2003

THIS PAGE INTENTIONALLY LEFT BLANK 


\section{NATIONAL NUCLEAR SECURITY ADMINISTRATION NEVADA OPERATIONS OFFICE NEPA ENVIRONMENTAL EVALUATION CHECKLIST}

\begin{tabular}{|l|l|}
\hline FOLLOW ATTACHED PROCEDURES FOR COMPLETING CHECKLIST & Date \\
A. Project/Activity Tite (Attach a brief description of proposed project) & $11 / 27 / 02$ \\
\cline { 2 - 3 } CAUs $176,346,350,351 \&$ 352: Housekeeping Sites Closure/Cleanup Activities &
\end{tabular}

Project Location

NTS, Various Areas

NNSAVNV Line Management Organization

Environmental Restoration Divislon

Anticipated Start Date

January 8, 2003

ENVIRONMENTAL CONSIDERATIONS: If any phase of the project/activity involves any of the following considerations, check yes and explain in project description. See NV-16A for consideration guidelines and examples.

\begin{tabular}{|c|c|c|c|c|c|c|c|}
\hline Consideration & Yes & No & Unk & Consideration & Yes & No & Unk \\
\hline 1. Noise & $\mathrm{x}$ & & & 10. Liquid Effluents & & $\mathrm{X}$ & \\
\hline 2. Air Emissions & & $\mathbf{x}$ & & 11. Underground Stcrage/Septic Tanks & & $\mathrm{x}$ & \\
\hline 3. Explosives & $\mathrm{x}$ & & & 12. Utility Systems (PCBs) & $\mathrm{X}$ & & \\
\hline 4. Petroleum/Fuel Storage/Use & $\mathrm{x}$ & & & 13. Environmental Restoration Site & $\mathrm{x}$ & & \\
\hline 5. Pesticide/Herbicide Use & & $\mathbf{X}$ & & 14. Change in Existing Drainage Pattern & & $\mathrm{x}$ & \\
\hline 6. Hazardous/Toxic Substances & $\bar{x}$ & & & 15. Surface Disturbance/Excavalion & $\bar{x}$ & & \\
\hline 7. Solid Waste & $\bar{x}$ & & & 16. Cultural/Historic Resources & & & $\mathrm{x}$ \\
\hline 8. Mixed Waste & & $\mathrm{x}$ & & 17. Biological/Tortoise Resources & & $\mathrm{X}$ & \\
\hline 9. Radioactive Materials & $\mathrm{X}$ & & & & & & \\
\hline
\end{tabular}

DO NOT TYPEOR WRITE BELOW THIS LINE, FOR ESHD USE ONLY.

B. Is the projectlactivity included in the final NTS EIS and the ROD or other NEPA document?

Yes $\mathrm{X}$ (complete Sections C, D, and E) No (complete Sections D, E, and F)

C. This project/activity is included in the NTS EIS/ROD (or other NEPA document) under the following section and page number:

NTSIEIS Volume 1. Appendix A, A.3,1,3 - Environmental Restoration Program: Industrial Sites Project

D. Does the proposed project/activity require any local, state, or federal permits or notifications?

Yes $\mathrm{X} \quad$ No

E. Does the proposed projectlactivity relate to the FFCA or FFACO agreements?

Yes $\mathrm{X} \quad$ No

F. If, based on the project description and the preliminary environmental considerations noted above, the proposed action fits within a class of action listed in Subpart D of 10 CFR 1021, write in the space below, the paragraph number and short title from the appropriate table of contents of Subpart D, Appendix B, C, or D, for a CX, EA, or EIS. If the proposed action does not fit within any class of action, write "Not Listed" below.

\section{G. NEPA COMPLIANCE OFFICER DETERMINATION OR RECOMMENDATION:}

I have determined that the proposed action as described in item A above, has been adequately addressed for the purpose of NEPA in the NTS EIS. No further analysis or documentation is required pursuant to NEPA. 


\section{Project Description}

All CAUs are housekeeping sites, located at the Nevada Test Site (NTS). CAU 176 is located in Areas 5 and 6 and consists of four Corrective Action Sites (CASs). CAU 346 is located in Areas 8 and 10 and consists of 13 CASs. CAU 350 is located in Area 15 and consists of nine CASs. CAU 351 is located in Areas 18 and 30 and consists of six CASs. CAU 352 is located in Areas 19 and 20 and consists of 14 CASs.

The purpose of this project is to remove the various debris located at each CAS. A detailed list of the CASs and descriptions is provided in the FFACO (1996). Each CAU is listed below, with a brief description of the types of debris present in the CASs.

\section{CAU 176}

Includes two small vacuums, an automotive battery, oll can, 330-gallon tank filled with sanitary trash, and scrap wood and metal. The vacuums and possibly the wood and metal debris are radioactive.

\section{CAU 346}

The majority of debris at the CASs comprising CAU 346 appear to be drums filled with concrete that have electrical cables running out from the concrete and extending to various boreholes. Other debris include hoses, scrap metal, wood debris, cables, wire, etc. Portions of these wastes may be radioactive.

\section{CAU 350}

Wastes at these CASs include metal and wood debris, empty fuel cans, several tins that are labelled "Blasting Caps," a deteriorated dry cell battery, and a gas cylinder approximately $4 \mathrm{ft} \times 1 \mathrm{f}$. None of the wastes are radiological, but the blasting cap tins and gas cylinder may be hazardous or toxic.

\section{CAU 351}

The majority of debris at these CASs includes dry cell batteries. Other waste includes wood, metal and glass debris, and two rusted, green jeeps. Some of the waste may be radioactive and or hazardous/toxic.

\section{CAU 352}

Debris includes a transformer, wood, rusted metal pipes, PVC pipes, chunks of concrete, broken batteries, empty cans and drums, and lead pellets. There is also a black stained area at one of the locations.

\section{Environmental Considerations}

1. Noise levels: Elevated noise levels may result from the operation of a backhoe andior loader equipment. Personnel not directly involved with operation of this equipment will be kept back at least 15 feet while equipment is in use. The equipment operator will follow the instructions as directed in the Site Specific Health and Safety Plan.

3. Explosives; Several tins marked "Blasting Caps" will be exploded in place. The certified blaster will direct all operations while during this phase of the cleanup.

4. Petroleum/Fuel Storage Use: Heavy equipment on site will use petroleum fuel. No fuel will be stored on site outside of the equipment. Absorbent pads will be used if equipment appears to be leaking petroleum.

6. Hazardous/Toxic Substances: Several sites contain lead, PCB or TPH-impacted and/or sanitary waste and soil. No other chemical hazards are known to exist. Personnel will be required to follow the safety procedures outined in the Site Specific Health and Safety Plan and Job Hazard Analysis. Wastes will be disposed of either on the NTS or offsite, depending on analysis results. 
7. Solid Waste: Any solid waste that may be generated from project activities will be disposed of at the NTS in a designated landfili.

9. Radloactive Materials: Several sites are in a URMA, CA, or RMA. Radiologically-impacted soil and manufactured items in these areas may require disposal at either the Area 3 or Area 5 Radioactive Waste Management Site.

12. Utility Systems (PCBs): Two sites contain transformers which are potentially contaminated with PCBs. These will be disposed of as PCB waste if found to contain PCBs. Personnel will be required to follow the safety procedures outlined in the Site Specific Health and Safety Plan and Job Hazard Analysis.

13. Environmental Restoration Site: These sites are included in the FFACO as Corrective Action Unit 176, 346, $350,351$. and 352. (See Project Description).

15. Surface Disturbance/Excavation: No excavations are planned. Equipment will travel off-road on previously disturbed areas. Equipment will also be used to help remove surface debris.

16. Cultural/Historic Resources: Several sites contain equipment and debris of mining activities which occurred before the Nevada Test Site was removed from the public domain. These artifacts will be left undisturbed. 
CLOSURE RE PORT - CAU 346 Section: Appendix C

Revision : 0

Date: August 2003

THIS PAGE INTENTIONALLY LEFT BLANK 
CLOSURE RE PORT - CAU 346

Section: Distribution List

Revision : 0

Date: August 2003

\section{DISTRIBUTION LIST}


CLOSURE RE PORT - CAU 346

Section: Distribution List

Revision : 0

Date: August 2003

\section{THIS PAGE INTENTIONALLY LEFT BLANK}




\section{DISTRIBUTION LIST}

*Provide copy of initial distribution of all revisions; others receive NDEP-approved revision only.

\section{$\underline{\text { Nevada Division of Environmental Protection }}$}

Paul Liebendorfer

1(Controlled)*

Bureau of Federal Facilities

Division of Environmental Protection

333 W. Nye Lane, Room 138

Carson City, NV 89706-0866

Donald Elle

Bureau of Federal Facilities

1 (Controlled)*

Division of Environmental Protection

1771 East Flamingo Road, Suite 121-A

Las Vegas, NV 89119-0837

\section{U.S. Department of Energy}

Janet Appenzeller-Wing

1 (Uncontrolled)*

Environmental Restoration Division

U.S. Department of Energy

National Nuclear Security Administration

Nevada Site Office

P.O. Box 98518, M/S 505

Las Vegas, NV 89193-8518

Kevin Cabble

Environmental Restoration Division

1 (Uncontrolled)*

U.S. Department of Energy

National Nuclear Security Administration

Nevada Site Office

P.O. Box 98518, M/S 505

Las Vegas, NV 89193-8518

Sabrina Lawrence

1 (Controlled)*

Environmental Restoration Division

U.S. Department of Energy

National Nuclear Security Administration

Nevada Site Office

P.O. Box 98518, M/S 505

Las Vegas, NV 89193-8518 


\section{DISTRIBUTION LIST (continued)}

\section{U.S. Department of Energy (continued)}

U.S. Department of Energy

National Nuclear Security Administration

Nevada Site Office

Public Reading Facility

c/o Nuclear Testing Archive

P.O. Box 98521, M/S 400

Las Vegas, NV 89193-8521

U.S. Department of Energy

National Nuclear Security Administration

Nevada Site Office

Technical Library

P.O. Box 98518

Las Vegas, NV 89193-8518

U.S. Department of Energy

Office of Scientific and Technical Information

P.O. Box 62

Oak Ridge, TN 37831-0062

\section{$\underline{\text { Bechtel Nevada }}$}

Correspondence Control

Bechtel Nevada

P.O. Box 98521, M/S CF008

Las Vegas, NV 89193-8521

Environmental Management Library

Bechtel Nevada

P.O. Box 98521, M/S NLV080

Las Vegas, NV 89193-8521

Kevin Campbell

Bechtel Nevada

P.O. Box 98521, M/S NTS306

Las Vegas, NV 89193-8521

Wayne Johnson

Bechtel Nevada

P.O. Box 98521, M/S NLV080

Las Vegas, NV 89193-8521
1 (Controlled) \&

1 (Uncontrolled)

1 (Uncontrolled) 


\section{DISTRIBUTION LIST (continued)}

\section{$\underline{\text { Bechtel Nevada (continued) }}$}

Patrick Morris

1 (Uncontrolled)*

Bechtel Nevada

P.O. Box 98521, M/S NTS306

Las Vegas, NV 89193-8521

Steve Nacht

1 (Uncontrolled)*

Bechtel Nevada

P.O. Box 98521, M/S NTS306

Las Vegas, NV 89193-8521

Reed Poderis

1 (Uncontrolled)*

Bechtel Nevada

P.O. Box 98521, M/S NTS306

Las Vegas, NV 89193-8521

Allison Urbon

1 (Uncontrolled)*

Bechtel Nevada

P.O. Box 98521, M/S NTS306

Las Vegas, NV 89193-8521

Shaw Environmental, Inc.

FFACO Coordinator

1 (Controlled)

Shaw Environmental

P.O. Box 93838, M/S 439

Las Vegas, NV 89193-3838

John Stokowski

1 (Uncontrolled)*

Shaw Environmental

P.O. Box 93838, M/S 439

Las Vegas, NV 89193-3838

\section{$\underline{\text { State of Nevada }}$}

Manager, Northern Nevada

1 (Uncontrolled)

FFACO Public Reading Facility

Nevada State Library and Archives Federal Publications

100 North Stewart Street

Carson City, NV 89701-4285 
CLOSURE RE PORT - CAU 346

Section: Distribution List

Revision : 0

Date: August 2003

\section{THIS PAGE INTENTIONALLY LEFT BLANK}

